\title{
A polarized atomic hydrogen beam
}

\author{
N. Chan ${ }^{1}$, D.M. Crowe ${ }^{1}$, M.S. Lubell ${ }^{1}$, F.C. Tang ${ }^{1}$, A. Vasilakis ${ }^{1}$, \\ F.J. Mulligan ${ }^{2}$, and J. Slevin ${ }^{2}$ \\ ${ }^{1}$ Department of Physics, City College of CUNY, New York, NY 10031, USA \\ ${ }^{2}$ Department of Experimental Physics, St. Patrick's College, Maynooth, Co. Kildare, Ireland
}

Received 21 July 1988

We describe the design and operating characteristics of a simple polarized atomic hydrogen beam particularly suitable for applications to crossed beams experiments. In addition to experimental measurements, we present the results of detailed computer models, using Monte-Carlo ray tracing techniques, optical analogs, and phase-space methods, that not only provide us with a confirmation of our measurement, but also allow us to characterize the density, polarization, and atomic fraction of the beam at all points along its path. As a subsidiary result, we also present measurements of the relative and absolute efficiencies of the V/G Supavac mass analyzer for masses 1 and 2.

PACS: $07.77 ; 35.10 . \mathrm{D} ; 35.80$

\section{Introduction}

The uniquely simple structure of atomic hydrogen makes it an ideal system for the theoretical and experimental study of fundamental physics. Whether used as a probe of the weak interaction [1], as a testing ground for quantum electrodynamics [2], as a laboratory for metrology [3] and the determination of the values of fundamental constants [4], or as a target for the study of basic atomic collisions [5-8], the hydrogen atom has continued to play a central role in our attempt to understand physical phenomena at their most basic level. As a consequence, much effort has been devoted to the development of atomic hydrogen beams, both ground state and excited state, both polarized and unpolarized [9-16]. Since the late 1960 's a large fraction of this effort has been directed toward the production of polarized proton, deuteron, and $\mathrm{H}^{-}$beams for applications to nuclear and particle physics [17]. Recently, substantial attention has also been given to the development of extremely cold, intense neutral beams [14] for use as internal targets in high-energy storage rings, as polarized proton sources for accelerator injection, and as the central element in hydrogen masers for time and frequency standards. Many of the hydrogen beams described in the literature, whether polarized or unpolarized, are large, complex, and costly devices that are not particularly suitable for small scale laboratory physics. In this paper we describe the characteristics of a relatively compact and inexpensive polarized ground-state atomic hydrogen beam that we developed for crossed-beams studies of low-energy electron-hydrogen scattering. We present the results of detailed computer modeling of the beam, as well as measurements of operating parameters.

The principal properties of a polarized hydrogen beam comprise its dissociation fraction or purity, its useful density and flux, and its polarization or state selection parameter. Such factors as its operational duty factor, its long and short-term stability, and its ease of operation are clearly important also. All of these characteristics depend upon the method used for producing the atoms, the technique employed for polarizing the atoms, and the specific details of the beam-line geometry.

Ground-state beams of atomic hydrogen are almost universally generated through the dissociation of hydrogen molecules by dc (Wood's) [18], rf, or microwave discharges [9], or alternatively by thermal methods that employ high-temperature tungsten or tantalum ovens $[6,19]$. Regardless of the method chosen, each beam design must confront vacuum needs, choice of materials, heating or cooling require- 
ments, and the geometry of skimmers and collimators, all of which have a direct bearing on the densities, fluxes, and dissociation fractions that will be achieved, in addition to the overall performance reliability of the beam. Often the choice of source technique will be dictated by the specific requirements of the experimental application.

In spite of their more specialized application, polarized hydrogen beams have been the subject of extensive development, although most of the attention they have received has been directed toward their use in polarized proton sources for high-energy and nuclear physics, as we have already noted. For these purposes, the spin of the proton is of paramount importance, and hence the neutral beams from which they derive must have a nuclear as well as an electronic polarization. The methods used to achieve the nuclear polarization vary depending upon whether metastable beams or ground state beams are the starting points. In virtually all cases, however, spin filters involving rf transitions are employed. By contrast, for our application, where the electron spin is of prime importance, we elected not to incorporate any rf spinfiltering, at the loss of some polarization at low magnetic field, but with the consequent gain in simplicity and beam intensity.

In Sect. II of the paper we review the experimental details of the hydrogen beam line including the rf source, the hexapole state selector, the Stern-Gerlach polarimeter and the quadrupole beam analyzer. In Sect. III we discuss the operating principles of the beam and the techniques we employed to determine its salient characteristics of polarization, density, and dissociation fraction. In Sect. IV we explore three methods of computer modeling of the beam including Monte-Carlo ray tracing, an optical approach that treats the hexapole magnet as a thick lens, and a Cartesian phase space technique that yields approximate information for axially symmetric beams. Finally, in Sect. V we discuss the results of our measurements, summarize the properties of the beam, and briefly compare our beam with several other polarized hydrogen beams developed elsewhere.

\section{Experimental design}

\section{A. Overview}

The beam line comprises five stainless steel vacuum chambers separated from each other by collimating apertures or conductance limiting tubes, as shown in Fig. 1. The locations and critical dimensions of the components and apertures encountered by the hydrogen beam are shown schematically in Fig. $2 a$ and b.

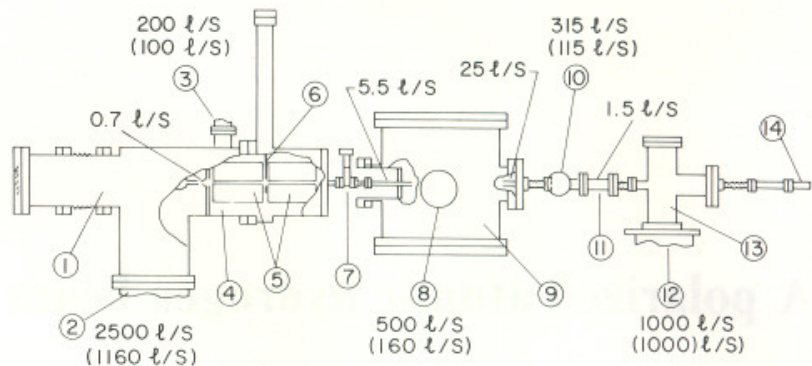

Fig. 1. Vacuum chamber layout of beam line showing conductances between chambers for molecular hydrogen, typical pressures under operating conditions, and effective speeds of pumping modules for both hydrogen and air, the latter enclosed in parentheses. The numbered elements shown are (1) hydrogen source chamber, (2) diffusion pump port, (3) turbomolecular pump port, (4) hexapole chamber, (5) hexapole magnets (6) beam chopper, (7) beam-line gate valve, (8) ion pump port, (9) interaction chamber, (10) ion pump port, (11) Stern-Gerlach magnet, (12) cryopump port, (13) quadrupole/dump chamber, and (14) quadrupole mass analyzer. Not shown are bypass pumping lines and valves between the hexapole and hydrogen source chambers and between the quadrupole and interaction chambers. Note that conductances for air may be obtained by multiplying the hydrogen conductances by a factor of 0.27

High-purity hydrogen, having entered the source through a heated palladium finger, is dissociated by an rf discharge in a water-cooled Pyrex tube [9]. The atoms and molecules effusing from the $1 \mathrm{~mm}$ diameter nozzle of the source are formed into a beam by a stainless steel skimmer, $1.4 \mathrm{~mm}$ in diameter, located $16.5 \mathrm{~mm}$ away and mounted on a stainless steel bulkhead. Slightly further downstream, $25.4 \mathrm{~mm}$ from the skimmer, the beam enters a pair of state-selecting hexapole magnets [20] each $152 \mathrm{~mm}$ long and each having a pole gap of $6.4 \mathrm{~mm}$. The magnets, which transmit atoms in the $m_{S}=+\frac{1}{2}$ state and deflect those in the $m_{S}=-\frac{1}{2}$ state, as described in Sect. IIIC, are separated by a gap of $19 \mathrm{~mm}$ to facilitate pumping. A beam chopper, driven by a stepping motor that is capable of modulating the beam at a maximum frequency of $10 \mathrm{~Hz}$ with typical open and closed times of $50 \mathrm{~ms}$ each, is mounted in the gap between the two magnets. The position of the hexapole pair is chosen on the basis of a computer analysis that optimizes the density of the beam at the interaction region for a room-temperature $v^{2}$-Maxwellian velocity distribution, as described in Sects. III and IV.

Upon leaving the downstream magnet, the beam first passes through a small gate valve that serves to isolate the source and magnet chambers from the rest of the beam line and then through a small conductance limiting aperture and tube around which is wound a spin-guiding solenoid, as shown in Fig. 2 b. The solenoid, together with two additional ones mounted inside the interaction chamber, serves to transport the atoms into the interaction region in 

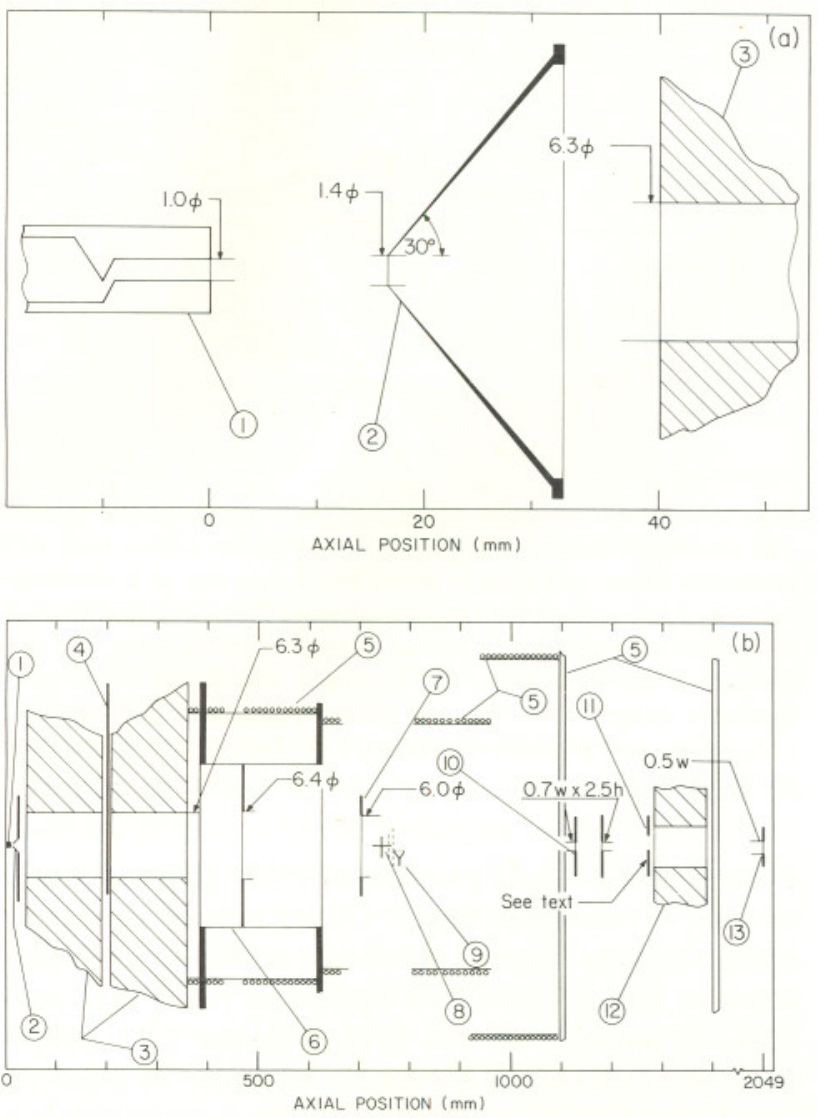

Fig. 2(a). Schematic diagram of beam-line components in the source region showing locations and critical dimensions of (1) nozzle, (2) skimmer, and (3) hexapole magnet entrance. All dimensions shown are in $\mathrm{mm}$; (b) Schematic diagram of major components of beam line showing locations and critical dimensions of (1) nozzle, (2) skimmer, (3) hexapole magnets, (4) beam chopper, (5) spin guiding magnet field coils (transverse rotator prior to Stern-Gerlach magnet not shown), (6) refrigerated tube and aperture, (7) interaction region beam collimator, $(8)$ crossed beams intersection point, (9) channel electron multiplier, (10) polarimeter slits, (11) SternGerlach entrance aperture, (12) Stern-Gerlach magnet, and (13) QMA entrance slit. All dimensions shown are in $\mathrm{mm}$

such a manner that the spins are adiabatically rotated [6] and maintained either parallel or antiparallel to the direction of the beam by the $100 \mathrm{mG}$ longitudinal magnetic field that is present at the center of the interaction region. A circular aperture $6.0 \mathrm{~mm}$ in diameter and located $38 \mathrm{~mm}$ upstream from the center of the interaction region serves to define the dimension of the beam.

At the exit of the interaction chamber, the beam again passes through a conductance limiting tube and aperture as shown in Fig. 2b. Magnetic guide fields (not shown in the figure) again serve to adiabatically rotate the atomic spins, this time into the transverse orientation, in preparation for polarization analysis by a Stern-Gerlach dipole magnet [21]. In front of the Stern-Gerlach polarimeter are a pair of slits each $0.7 \mathrm{~mm}$ wide by $9.5 \mathrm{~mm}$ high, that are externally movable while the system is under vacuum. For purposes of polarimetry, where scattering from the Stern-Gerlach pole tips poses a problem, the slits are inserted; for purposes of beam monitoring, where maximum signal strength is of paramount importance, however, the slits are removed. Since the dimension of the collimator associated with the Stern-Gerlach magnet is only of order $2.5 \mathrm{~mm}$ at a location where the beam diameter is approximately $10 \mathrm{~mm}$, the vacuum chamber directly upstream of the entrance to the polarimeter effectively serves as a "dump" for at least half of the hydrogen beam.

The atoms and molecules that are accepted by the Stern-Gerlach unit enter the final beam line chamber which contains a Vacuum Generators Supavac quadrupole mass analyzer (QMA). Immediately in front of the QMA is a third movable slit $0.5 \mathrm{~mm}$ wide that is also externally accessible while the system is under vacuum. As with those upstream, the QMA slit is inserted for polarization measurements and removed for normal beam monitoring. The QMA and its slit are both mounted in a vacuum pipe that is attached to a horizontal translator, thereby permitting the profile of the beam transmitted through the Stern-Gerlach magnet to be scanned as part of the polarimetry procedure, as described in Sect. III.

For each section of the beam line, vacuum pumps were chosen that met the specific experimental requirements of the particular section and also minimized the cost per unit pumping speed. The hydrogen source chamber is pumped by a high-speed oil diffusion pump [22] and the hexapole chamber, by a turbomolecular pump [23], with each backed by a dedicated mechanical pump. Immediately beyond the hexapole chamber and mounted directly in the beam line is a low-conductance freon-cooled tube that protects sensitive downstream pumps and experimental components from any accidental upstream release of hydrocarbon contamination. (In this context, however, it should be noted that in more than four years of operation, there has not been a single instance of such a release.) For purposes of meeting ultra-high vacuum requirements and maintaining a hydrocarbon-free environment, the interaction chamber is pumped by an ion pump [24] designed for hydrogen use. An optically opaque "spiral staircase" baffle effectively eliminates charged particle backgrounds resulting from ion and electron backstreaming. The Stern-Gerlach region is pumped by another ion pump [25], also designed for hydrogen use, but in this case in an unbaffled configuration. At the QMA location, where greater precaution must be taken against ion backgrounds, a closed loop helium cryopump [26] is used. 


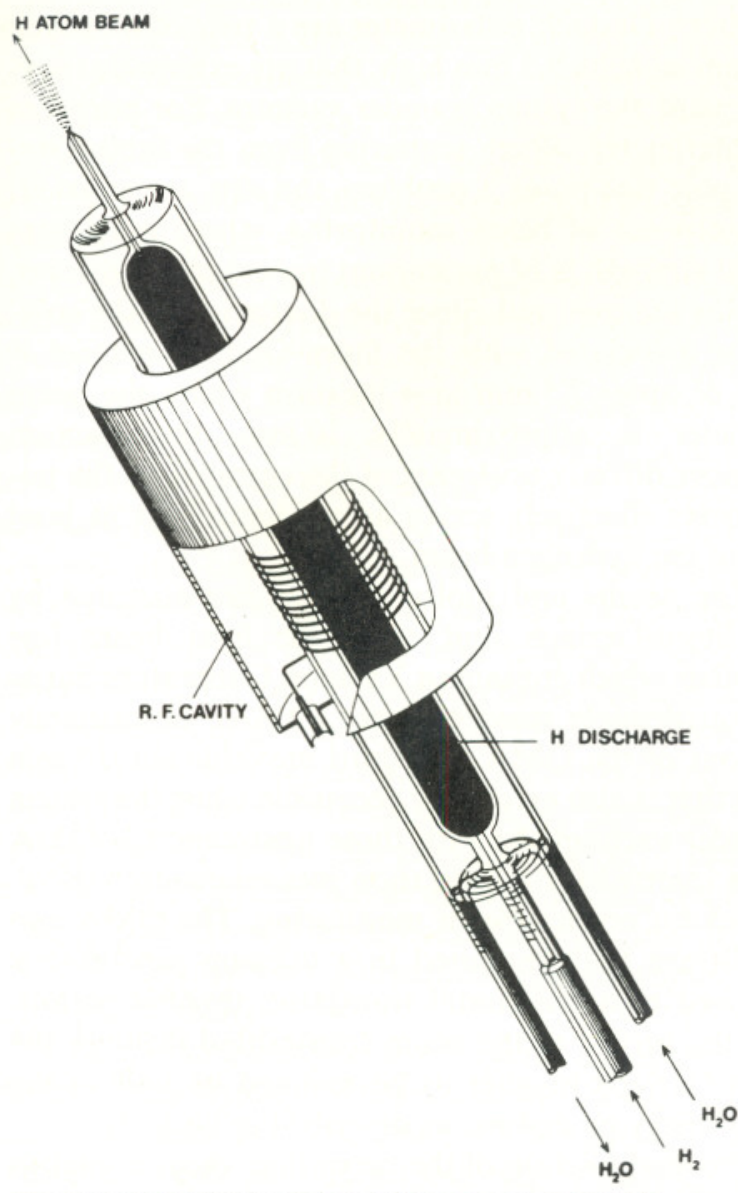

Fig. 3. Artist's representation of the rf hydrogen source

\section{B. RF hydrogen source}

Fabricated in Scotland and essentially a duplicate of a source previously described [9] (a version of which is now available commercially) [27], the rf dissociator utilizes a water cooled Pyrex discharge tube operating in a coaxial cavity with $15 \mathrm{~W}$ of power at $35 \mathrm{MHz}$. With standard precautions taken to avoid ground loops in sensitive amplifiers throughout the experimental apparatus, the source, shown schematically in Fig. 3, produces minimal $\mathrm{rf}$ interference. The stability of the discharge and the degree of dissociation are critically dependent upon both the steady flow of bubble-free cooling water and the cleanliness of the Pyrex surface. The former condition is easily achieved if the cooling water is turned on several hours before the source is to be used. The latter condition is met through careful cleaning of the Pyrex tube (as described in [9] prior to installation of the source and through the use of a heated palladium leak to provide high-purity hydrogen during operation.

High purity hydrogen gas is provided by the gas handling system shown in Fig. 4. Pre-purified grade

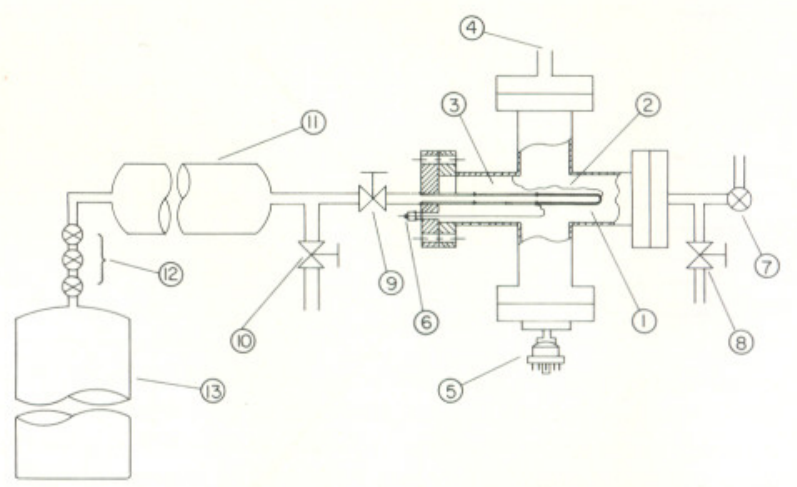

Fig. 4. Schematic diagram of the hydrogen gas handling system. The numbered elements shown are (1) hydrogen ballast tank, (2) palladium finger, (3) glass to metal break, (4) exit to rf discharge volume (5) thermocouple vacuum gauge, (6) electrical feedthrough for palladium heating (7) source chamber bypass valve, (8) dry nitrogen admittance valve, (9) hydrogen admittance valve, (10) hydrogen purge valve, (11) low-pressure hydrogen holding tank, (12) valves and pressure regulators, (13) high-pressure hydrogen gas cylinder

hydrogen at a supply pressure of $15-20$ psig is allowed to enter a palladium finger. Under normal operating conditions, the finger is resistively heated to a temperature of about $150^{\circ} \mathrm{C}$, an operating point that produces a pressure of $400 \mathrm{mTorr}$ in the 0.31 ballast cross piece shown in the figure. In order to preclude contamination of the palladium finger, care is taken to maintain the hydrogen supply line at a positive pressure with respect to atmosphere whether or not the source is in use.

\section{Hexapole magnet}

The permanent hexapole magnet, a cross section drawing of which is shown in Fig. 5, consists of two identical units, each $152 \mathrm{~cm}$ long and separated from each other by a gap of $19 \mathrm{~mm}$. The design of the magnet is based upon that used in the first polarized electron source installed at the Stanford Linear Accelerator Center [20] and produces a pole-tip field strength $H\left(R_{M}\right)$ of $0.79 \mathrm{~T}$ with a pole gap $2 R_{M}$ of $6.4 \mathrm{~mm}$. An alternate approach that achieves similar operating characteristics, still with permanent magnet construction, involves the use of samarium-cobalt elements that serve as integral drivers and pole tips $[28,29]$.

\section{Interaction region}

The crossed beams interaction region contains many components that are specific to the spin-tagged electron-hydrogen collisions studies being carried out and 


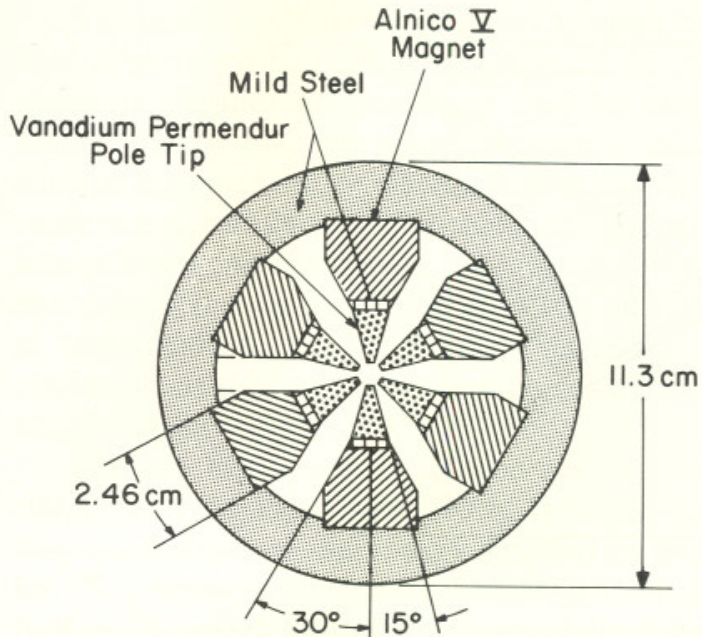

Fig. 5. Scale drawing of the hexapole magnet in cross sectional view

are not germane to this paper. The only elements that are pertinent to the hydrogen beam are the apertures and spin-guiding field coils shown in Fig. 2 b, the dimensions and operating conditions of which are given in the figure. At the interaction region center, the influence of the earth's magnetic field is maintained below $10 \mathrm{mG}$ with the use of a single layer of $\mu$-metal shielding $1.5 \mathrm{~mm}$ thick. The shielding, in the shape of a capped cylinder, located just inside the vacuum chamber walls, is equipped with coils that permit the $\mu$-metal to be degaussed in situ, a procedure that obviates the need for any annealing of the material. When the guide fields are on, the magnetic field at the center of the interaction chamber is approximately $100 \mathrm{mG}$ oriented either parallel or antiparallel to the direction of the hydrogen beam line.

\section{E. Stern-Gerlach magnet}

The Stern-Gerlach magnet [21], employed for polarimetry measurements, consists of an electromagnetic driver located outside the vacuum system and lowcarbon magnet iron pole faces placed inside the vacuum system. Of a standard "two wire" geometry, the pole faces, $101.6 \mathrm{~mm}$ long with a minimum horizontal gap of $3.18 \mathrm{~mm}$, are shown in cross section in Fig. 6, producing a field to gradient ratio of $\sim 6 \mathrm{~mm}$. The entrance to the magnet is masked by a collimating aperture $2.5 \mathrm{~mm}$ in height in combination with a single knife edge that extends horizontally into the gap a distance of $0.7 \mathrm{~mm}$ from the leading edge of the convex pole tip. Such a configuration on one hand provides reasonable throughput during ordinary beam intensity monitoring and on the other hand, in conjunction with a pair of movable upstream slits,

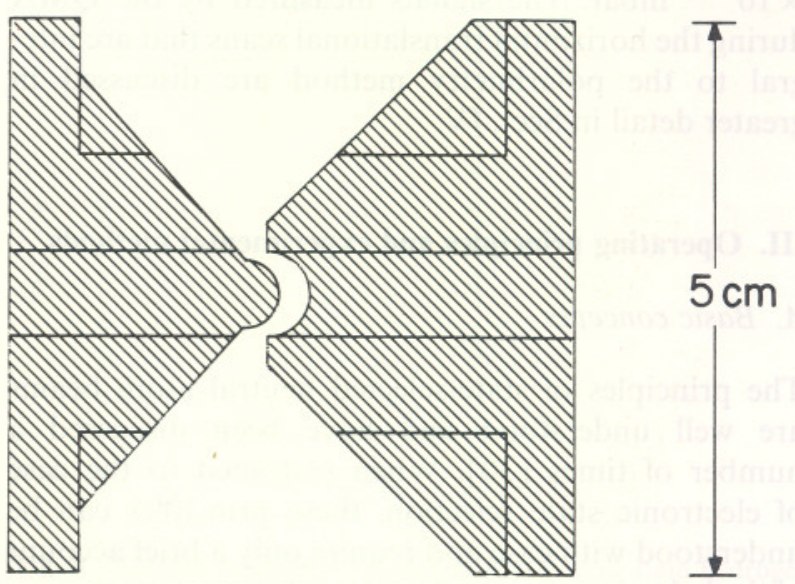

Fig. 6. Scale drawing of the Stern-Gerlach magnet in cross sectional view

minimizes pole tip scattering during beam polarization measurements. In the latter application, the magnet is energized with a maximum current of $5.5 \mathrm{~A}$, which produces a pole tip field strength $H(\mathfrak{I})$ of approximately $0.33 \mathrm{~T}$. For beam intensity monitoring, the energizing dc current is turned off, the magnet is degaussed, and the pair of upstream slits, $0.5 \mathrm{~mm}$ wide by $9.5 \mathrm{~mm}$ high, are removed from the beam path.

\section{F. Quadrupole mass analyzer}

The quadrupole mass analyzer (QMA) is a Vacuum Generators Supavac model that uses a Faraday cup and a high-gain amplifier for ion detection. During polarization measurements, the analyzer entrance is masked by a slit $0.5 \mathrm{~mm}$ wide. With the slit removed, as is the case during beam intensity monitoring, the analyzer presents a circular entrance aperture $6.4 \mathrm{~mm}$ in diameter, a dimension that effectively characterizes the entrance height when the slit is in place.

The analyzer, having a maximum sensitivity (defined by a signal to noise ratio of $2: 1$ ) of $2 \times 10^{-11}$ mbar, produces typical readings of $3.0 \times 10^{-10} \mathrm{mbar}$ and $1.3 \times 10^{-10}$ mbar respectively for $\mathrm{H}_{1}$ and $\mathrm{H}_{2}$ detection when the rf source is operating under standard conditions and the beam line slits are removed for intensity monitoring. When the rf power is turned off, the $\mathrm{H}_{1}$ signal drops to $3 \times 10^{-11} \mathrm{mbar}$ and the $\mathrm{H}_{2}$ signal rises to $4.3 \times 10^{-10}$ mbar. In the polarization measurement mode, with the slits inserted and the lateral position of the QMA adjusted for maximum signal, the typical $\mathrm{H}_{1}$ and $\mathrm{H}_{2}$ readings for $\mathrm{rf}$ power on are $4 \times 10^{-11} \mathrm{mbar}$ and $5 \times 10^{-11} \mathrm{mbar}$ respectively. With the rf power turned off the corre- 
sponding readings are $1 \times 10^{-11} \mathrm{mbar}$ and 1.3 $\times 10^{-10}$ mbar. The signals measured by the QMA during the horizontal translational scans that are integral to the polarimetry method are discussed in greater detail in Sect. IV.

\section{Operating principles and experimental methods}

\section{A. Basic concepts}

The principles of state-selected neutral atom beams are well understood and have been discussed a number of times [30]. When restricted to the case of electronic state selection, these principles can be understood with ease and require only a brief account of the behavior of an atom in an inhomogeneous magnetic field. As the Breit-Rabi diagram of Fig. 7 demonstrates for ground state atomic hydrogen, the hyperfine substates, characterized at low magnetic field by the total angular momentum quantum numbers $F$ and $m$, are split and regrouped at high magnetic field according to the electronic and nuclear spin quantum numbers $m_{J}$ and $m_{I}$. At fields sufficiently high to decouple electron and nuclear spins (but not high enough to decouple spin and orbital angular momentum), the magnetic energy of an atom, $\Delta W$, in an external magnetic field $\mathbf{H}$ is given by [31]

$$
\Delta W \approx \mu_{B} H m_{J} g_{J}+A m_{I} m_{J},
$$

where $\mu_{B}$ is the Bohr magneton, $g_{J}$ is the Landé $g$ factor, and $A$ is the hyperfine contact energy, with the nuclear multipole interactions with the external field being neglected. The atom then has an effective magnetic moment $\mu_{\text {eff }}$ given by

$\mu_{\mathrm{eff}} \approx-\mu_{B} m_{J} g_{J}$.

In a non-uniform magnetic field, a ground state hydrogen atom will thus experience a force $\mathbf{F}$ given by

$\mathbf{F}=-\nabla(\Delta W) \approx \mp \mu_{B} \nabla H$,

for $m_{J}=m_{S}= \pm \frac{1}{2}$, where $g_{J}$ is taken to be equal to 2. From (2) it is obvious that in principle any inhomogeneous magnetic field will create a spatial separation between atoms in the $m_{S}=+\frac{1}{2}$ state and those in the $m_{S}=-\frac{1}{2}$ state. In practice, two field geometries afford the greatest advantage. For polarized beam generation, hexapole magnets provide the geometry of choice, while for polarimetry applications, both hexapole and two-wire dipole fields can be used with success.

In the former instance, the hexapole field, which has a radial dependence given by [30]

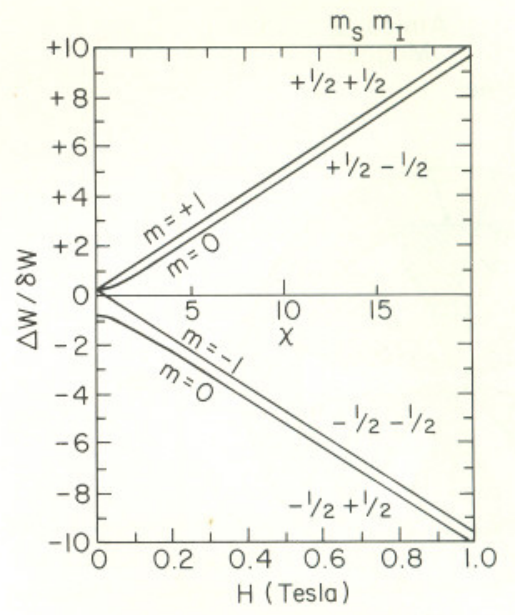

Fig. 7. Breit-Rabi diagram of ground state hydrogen

$H(r)=\frac{r^{2}}{R_{M}^{2}} H\left(R_{M}\right)$

with $r$ the radial coordinate, $2 R_{M}$ the pole gap, and $H\left(R_{M}\right)$ the pole tip field strength, causes the $m_{S}=+\frac{1}{2}$ atoms to oscillate with elliptical trajectories about the beam axis and the $m_{S}=-\frac{1}{2}$ atoms to be deflected out of the beam entirely. Thus for $m_{S}=+\frac{1}{2}$ atoms, a hexapole state-selecting magnet preserves the cylindrical qualities of the entering beam; moreover, it acts as a lens for these atoms, albeit with chromatic aberration, as has been discussed in detail elsewhere $[30,32]$. For a given field strength, the length of the magnet, and its position relative to both the atom source and the downstream interaction point therefore can be chosen to optimize the atom density at the interaction point. In the case of the atomic hydrogen beam described in this paper, the field strength of the hexapole was actually chosen to be the highest that could be achieved with permanent Alnico magnetic drivers, the objective being generation of maximum $m_{S}=+\frac{1}{2}$ state selection. The pole gap dimension was then chosen together with the remaining parameters to maximize the atom density for a $6.0 \mathrm{~mm}$ beam diameter at the interaction point, in accordance with the computer projections described in Sect. IV.

By contrast with the upstream polarizer, the downstream Stern-Gerlach polarimeter was designed around a dipole geometry, an approach that permits detailed scanning of the beam profile. In addition, while the hexapole uses a permanent magnet design, which minimizes cost and space, the Stern-Gerlach polarimeter employs an electromagnet approach, which allows the device to be demagnetized, an essential condition for both beam intensity monitoring and centroid determination. In the polarimetry process, the latter is an integral step, as explained in Sect. IIIC. 
Throughout the preceding description, the highfield approximation has been assumed for magnetic substates. While this condition may be met in the Stern-Gerlach magnet, given its narrow acceptance slit and its operating pole tip field strength, the assumption certainly needs further justification for the hexapole magnet. Moreover, an approach is required that relates the state selection achieved and measured at high fields to the electronic polarization that is applicable at the low fields characteristic of the interaction region.

For any field strength, $H$, the energy, $\Delta W$, associated with a magnetic substate is found from the diagonalization of the energy matrix. In particular, for ${ }^{2} S_{1 / 2}$ terms, which comprise the ground state hyperfine ${ }^{2} S_{1 / 2}$ multiplet of hydrogen, the secular determinant is of second order, and $\Delta W$ can be shown to be [31]

$\Delta W=-\frac{\delta W}{2(2 I+1)} \pm \frac{\delta W}{2} \sqrt{1+\frac{4 m}{2 I+1} \chi+\chi^{2}}$

for $m=+\left(I+\frac{1}{2}\right), \quad+\left(I-\frac{1}{2}\right), \ldots, \quad-\left(I-\frac{1}{2}\right), \quad-\left(I+\frac{1}{2}\right)$, where $I$ is the nuclear spin, $\delta W=(2 I+1) A / 2$ is the zero field hyperfine structure splitting between the $F=I-\frac{1}{2}$ and $F=I+\frac{1}{2}$ and $\chi$ is a dimensionless field variable defined by

$\chi \equiv g_{J} \mu_{B} H / \delta W$,

the nuclear multipole interactions with the external field being neglected throughout. Now the effective magnetic moment of the atom must be defined through the relation

$\mu_{\mathrm{eff}}=-\frac{\partial(\Delta W)}{\partial H}$.

For the maximum and minimum values of $m$; namely, $m= \pm\left(I+\frac{1}{2}\right), \mu_{\text {eff }}$ is independent of $\chi$ and is given by

$\mu_{\mathrm{eff}}\left[m= \pm\left(I+\frac{1}{2}\right)\right] \approx \mp \frac{1}{2} g_{J} \mu_{B}$.

For hydrogen, where $I=\frac{1}{2}$ and $g_{J} \approx 2,(8)$ reduces to

$\mu_{\mathrm{eff}}(m= \pm 1) \approx \mp \mu_{B}$.

For other values of $m, \mu_{\text {eff }}$ depends upon $\chi$ in a complex fashion, as suggested by Fig. 8 which illustrates the behavior of $\mu_{\text {eff }}$ as a function of $H$ or $\chi$ for the ground state of atomic hydrogen. As can be seen from the figure, the limiting values of $\mu_{\text {eff }}$ for $m=0$ are given by $\mu_{\text {eff }}=0$ for $\chi=0$ and $\mu_{\text {eff }}\left(m_{S}= \pm \frac{1}{2}\right) \rightarrow \pm \mu_{B}$ for $\chi \rightarrow \infty$.

Conventionally, the polarization $\mathbf{P}$ of a collection of electrons is defined [33] as the ensemble average of the expectation value of the Pauli spin operator $\sigma$ :

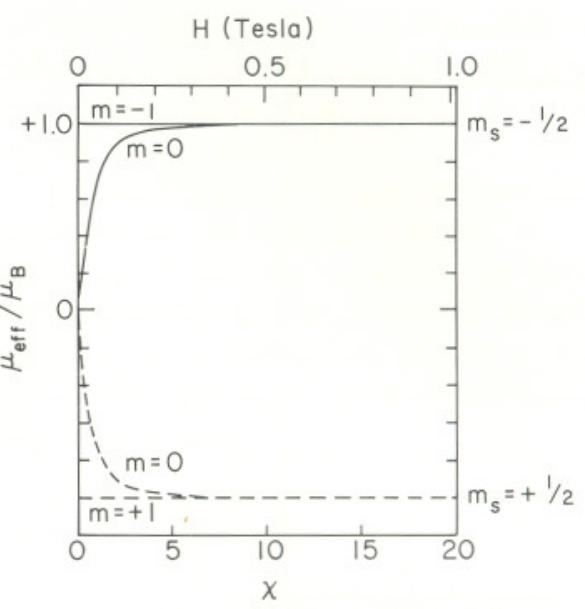

Fig. 8. Effective magnetic moments of the ground state hydrogen hyperfine multiplet as functions of magnetic field $H$ and field parameter $\chi$

$\mathbf{P}=\langle\boldsymbol{\sigma}\rangle$.

By virtue of the relationships

$\boldsymbol{\mu}=-g_{e} \mu_{B} \mathbf{S} / h=-g_{e} \mu_{B} \boldsymbol{\sigma} / 2$,

where $\mathbf{S}$ is the spin operator and $g_{e} \approx 2$ is the electron $\mathrm{g}$-factor, $\mathbf{P}$ can also be expressed as

$\mathbf{P}=-\left\langle\boldsymbol{\mu} / \mu_{B}\right\rangle$.

For an atomic beam which has undergone high-field state selection with $m_{S}=+\frac{1}{2}$ substates equally sharing one population and all $m_{S}=-\frac{1}{2}$ substates equally sharing another population, it is therefore reasonable to define a field-dependent quantity $f(\chi)$, conventionally called the hyperfine coupling function [30], by applying the concepts embodied in (12) to the expression for $\mu_{\text {eff }}$ derived from (5) and (7). Specifically, with P replaced by $f(\chi)$ and $\mu$ replaced by $\mu_{\text {eff }}$, we obtain the result

$f(\chi)=\frac{1}{2 I+1} \sum_{m=-I+\frac{1}{2}}^{I+\frac{1}{2}}\left(\mu_{\mathrm{eff}} / \mu_{B}\right)$,

which has the limiting values of $f(0)=1 /(2 I+1)$ and $f(\infty)=1$. For hydrogen, (13) reduces to

$f(\chi) \approx \frac{1}{2}\left[1+\frac{\chi}{\sqrt{1+\chi^{2}}}\right]$,

a graphical display of which is shown in Fig. 9. If at high-field, a state selection parameter $s$ is defined by

$s \equiv \frac{N_{+}-N_{-}}{N_{+}+N_{-}}$, 


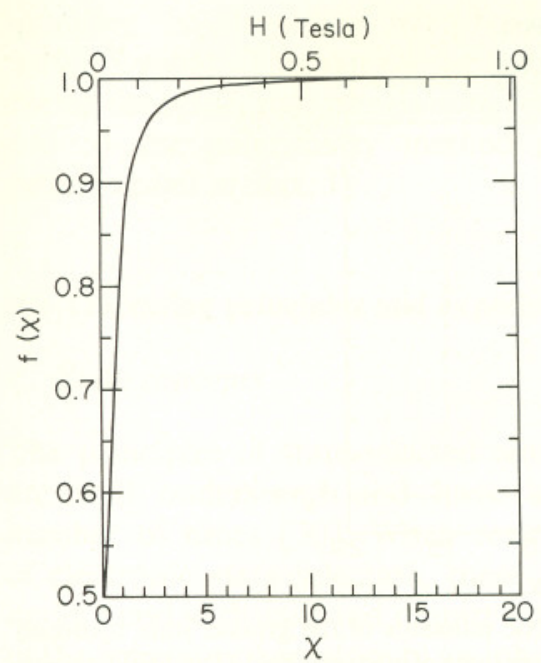

Fig. 9. Hyperfine coupling function $f(\chi)$

where $N_{+}$and $N_{-}$are respectively the number of atoms in the $m_{S}=+\frac{1}{2}$ and $m_{S}=-\frac{1}{2}$ states, then the electronic polarization $P(\chi)$ is given by

$P(\chi)=s f(\chi)$.

For the case in which the $m_{S}= \pm \frac{1}{2}$ substates have differing populations after high-field state selection, $P(\chi)$ must be calculated from the weighted average of $\left(\mu_{\mathrm{eff}} / \mu_{B}\right)$ over all the substates, the statistical weight, of course determined from the relative population of the respective substate in the usual fashion.

In carrying out the design of the beam and in determining its properties, we applied the principles developed in the foregoing paragraphs in two significant ways. First, using (6) and the radial reductions of (2) and (7), we developed the computer models described in Sect. IV to determine the behavior of the hydrogen beam as it traverses the hexapole and SternGerlach dipole magnets, with particular attention paid to tests of the validity of the high-field approximation. Second, from the results of the computer modeling, we related the high-field Stern-Gerlach polarization measurements to the value of the low-field polarization applicable at the interaction region. These applications will become clearer in the descriptions of the succeeding sections.

\section{B. Polarization determination}

From the concepts set forth in the preceding section, it is obvious that a dipole magnet with a cross section and orientation shown in Fig. 6 will cause a beam of atoms that is traveling outward from the plane of the figure to be deflected to the right or to the

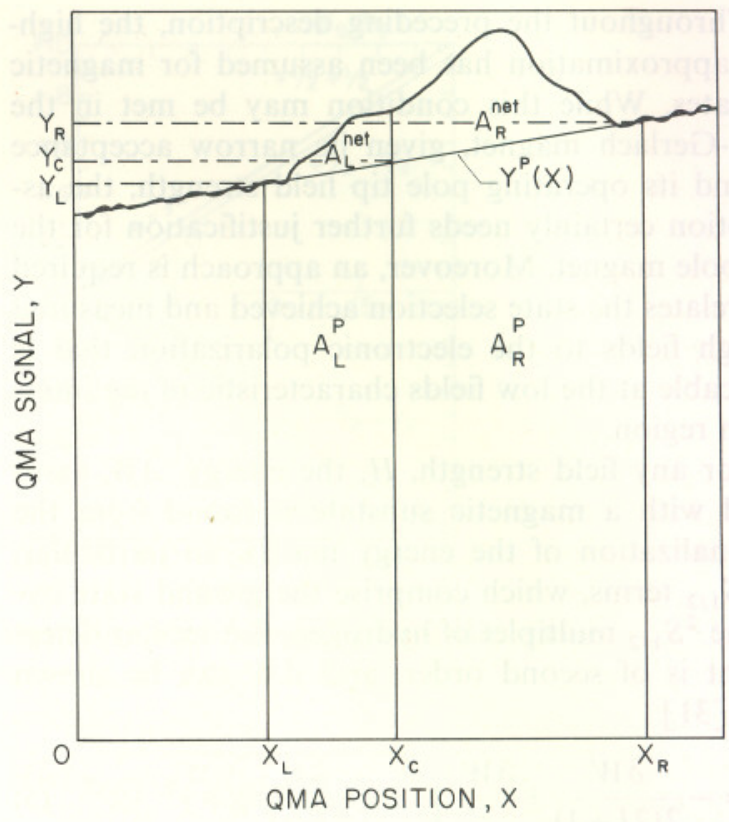

Fig. 10. Pictorial representation of the QMA profile used in the polarization determination, with symbols as defined in the text

left depending upon whether $\mu_{\text {eff }}$ is positive or negative respectively. In other words, atoms in the $m_{S}=$ $+\frac{1}{2}$ state will be deflected to the left while those in the $m_{S}=-\frac{1}{2}$ state will be deflected to the right. With the magnet power turned off and the pole tips degaussed, atoms in either state will pass through the unit undeflected.

The high-field polarization of the beam at the Stern-Gerlach magnet is thus determined as follows. From appropriate readings of the QMA and with appropriate settings of the source $\mathrm{rf}$ power, the net mass-one signal, is measured as the QMA is scanned horizontally across the beam. Based upon the beam profile when the Stern-Gerlach field is set at zero, a centroid $x_{C}$ is established, in terms of which a rightleft asymmetry can be defined for all non-zero settings of the Stern-Gerlach field. The high-field polarization is then given by the high-field limit of this asymmetry as the Stern-Gerlach field is steadily increased.

Figure 10 provides a pictorial representation of the profile generated when a motorized translator under the control of an LSI 11/23 computer drives the QMA horizontally across the beam in steps of $0.125 \mathrm{~mm}$. During acquisition of the polarization data, all three collimating slits are inserted into the horizontal position, as described in Sect. II.A. At each horizontal position, up to 9000 samples are taken of the QMA output voltage, with the actual number entered as input information to the data acquisition program. 
As illustrated in Fig. 10, the profile obtained with the rf source power on and the QMA tuned to mass one can be divided into four regions, two central ones on either side of the centroid containing signals related to both the beam and the molecular background, and two outer regions containing signals related to the molecular background alone. In all four regions, the background is found to vary linearly with position. Thus, in the central regions, the QMA signal can be characterized by three separate contributions - first, a pedestal due to dissociative ionization of the molecular background by the QMA electron bombarder; second, a portion (not labeled in the figure) due to dissociative ionization of the molecular component of the beam; and third, the net mass-one signal, which is the quantity of interest. The analysis procedure begins with the determination of the first two contributions and concludes with the subtraction of these from the total signal to generate the net massone signal.

At the outset of the procedure, the boundaries $x_{L}$ and $x_{R}$, between the central and outer regions on the left and right sides of the centroid respectively, are chosen by visual inspection. A standard regression analysis is then performed in the outer regions to determine the pedestal function $y^{P}(x)$, which enables the areal contributions of the pedestal, $A_{L}^{P}$ and $A_{R}^{P}$, to the left and right central regions to be calculated according to the prescriptions $A_{L}^{P}=\left(y_{L}^{P}+y_{C}^{P}\right)\left(x_{C}\right.$ $\left.-x_{L}\right) / 2$ and $A_{R}^{P}=\left(y_{R}^{P}+y_{C}^{P}\right)\left(x_{R}-x_{C}\right) / 2$. It should be noted that the variance calculated by the linear regression from the reduced chi-square is used as the variance of each point of the profile in all subsequent treatment of the data [34].

The determination of the areas $A_{L}^{D}$ and $A_{R}^{D}$, associated with contributions from molecules in the beam, requires the acquisition of three additional profiles corresponding to $\mathrm{rf}$ off with mass-one tuning $\left[\left(Q_{1}\right)^{\text {off }}\right]$, rf off with mass-two tuning $\left[\left(Q_{2}\right)^{\text {off }}\right]$, and $\mathrm{rf}$ on with mass-two tuning $\left[\left(Q_{2}\right)^{\text {on }}\right]$. In each case, the pedestal is subtracted from the total signal in accordance with the procedures described for the case of $\left[\left(Q_{1}\right)^{\text {on }}\right]$, thereby producing a net signal that is proportional to the molecular content of the beam for each case. As a consequence, it is easily seen that $A_{L}^{D}$ and $A_{R}^{D}$ are given by

$A_{L, R}^{D}=\frac{A\left[\left(Q_{2}\right)^{\mathrm{on}}\right]}{A\left[\left(Q_{2}\right)^{\mathrm{off}}\right]} A_{L, R}\left[\left(Q_{1}\right)^{\mathrm{off}}\right]$,

where $A\left[\left(Q_{2}\right)^{\text {on, off }}\right]=A_{L}\left[\left(Q_{2}\right)^{\text {on, off }}\right]+A_{R}\left[\left(Q_{2}\right)^{\text {on, off }}\right]$ and where all three profile areas are calculated from simple Riemann sums. Empirically it was found that $A_{L}^{D}$ and $A_{R}^{D}$ remained constant for at least a twelvehour period while the source was operating. Thus the $\left(Q_{1}\right)^{\text {off }},\left(Q_{2}\right)^{\text {off }}$, and $\left(Q_{2}\right)^{\text {on }}$ profiles were measured only once a day, and the values of $A_{L}^{D}$ and $A_{R}^{D}$ determined from them were applied to all $\left(Q_{1}\right)^{\text {on }}$ profiles measured that day.

Once $A_{L, R}^{P}$ and $A_{L, R}^{D}$ are determined, they are subtracted from the respective areas, $A_{L}$ and $A_{R}$, corresponding to the total signals in the left and right central regions of the $\left(Q_{1}\right)^{\text {on }}$ profile. In this way the net areas, $A_{L, R}^{\text {net }}$, are obtained, in terms of which a rightleft asymmetry, $\Delta_{H}$, can be constructed according to the prescription

$$
\begin{aligned}
\Delta_{H} & =\frac{A_{R}^{\text {net }}-A_{L}^{\text {net }}}{A_{R}^{\text {net }}+A_{L}^{\text {net }}} \\
& =\frac{\left(A_{R}-A_{R}^{P}-A_{R}^{D}\right)-\left(A_{L}-A_{L}^{P}-A_{L}^{D}\right)}{\left(A_{R}-A_{R}^{P}-A_{R}^{D}\right)+\left(A_{L}-A_{L}^{P}-A_{L}^{D}\right)} .
\end{aligned}
$$

It should be noted that throughout this discussion it has been assumed that the $Q_{1}$ and $Q_{2}$ signals and hence all the areas used in (18) are related to QMA readings obtained with the hydrogen beam chopper fixed in the open position.

The validity of the foregoing analysis rests strongly on the proper identification of the profile centroids that correspond to the true beam center when the Stern-Gerlach field is set at zero. Unless the hexapole magnet is perfectly aligned, it is clear that the centroids of the $\left(Q_{1}\right)^{\text {off }}$ signal and the determination of the true centroid of the net $\left(Q_{1}\right)^{\text {on }}$ signal for zero field thus requires that $x_{C}$ be varied until $\Delta_{H}$ is minimized. The value of $x_{C}$ so obtained then serves as the centroid for all calculations of $\Delta_{H}$ corresponding to nonzero values of the Stern-Gerlach field.

With $\Delta_{H}$ determined for a number of field settings, a plot can then be made of $\Delta_{H}$ as a function of the Stern-Gerlach driving current. As the current increases and $M_{S}= \pm \frac{1}{2}$ states are separated from each other further in space, $\Delta_{H}$ increases continuously until, under ideal conditions, the separation is complete, at which point $\Delta_{H}$ remains constant for all further increases in current. The saturation value $\Delta_{H}^{\max }$ is then taken as the high-field polarization of the analyzed portion of the beam.

\section{Density determination}

Information about the density of the hydrogen beam at the interaction region can be gleaned from several sources. Probably the least accurate determination, but by far the easiest one to make, results from the pressure increase, $\Delta p$, measured at the interaction chamber pump when the hydrogen beam chopper is opened and the beam is fully dumped in the interaction chamber. If the chamber is pumped with an effec- 
tive speed $S(l / s)$, then the number of atoms of gas $\mathrm{N}$ entering the chamber per unit time is given by [35]

$\frac{\mathrm{d} N}{\mathrm{~d} t}=\frac{2 S \Delta p}{k T}$,

where $T$ is the Kelvin temperature of the gas, $k$ is Boltzmann's constant, and the factor of two reflects recombination of the atoms prior to entry into the pump. Now for a radius $R_{H}$ characterizing the cylindrically symmetric, collimated beam at the interaction region, an average atom density $\bar{\rho}$ can be defined as

$\bar{\rho} \equiv \frac{1}{\pi R_{H}^{2}} \int_{0}^{R_{H}} \rho(r) 2 \pi r \mathrm{~d} r$,

where $\rho(r)$ is the atom density of the beam in the interaction region at a radius $r$. It should be clear that $\bar{\rho}$ and $\mathrm{d} N / \mathrm{d} t$ are related to each other by the expression

$\bar{\rho}=\frac{f}{\pi R_{H}^{2} \bar{v}} \frac{\mathrm{d} N}{\mathrm{~d} t}$,

where $\bar{v}$ is the average atom velocity in the interaction region, and $f$ is the fraction of atoms in the beam falling within a radius $R_{H}$. From (19) and (21) it then follows that $\bar{\rho}$ is given by

$\bar{\rho}=\frac{2 f S \Delta p}{\pi R_{H}^{2} \bar{v} k T}$.

It should be noted that $\bar{v}$ is not the customary velocity obtained from kinetic theory, since the hexapole magnet is a strongly chromatic lens. The only easy access to $\bar{v}$ is gained through computer modeling, as will be discussed in Sect. IVD. Of the other quantities in (22), all are known either by specification or measurement except for the fraction $f$ which depends upon the shape of the beam profile at the interaction region. As with $\bar{v}$, the experimental determination of $f$ cannot be made with ease, as a consequence of which the value must be calculated from the profiles generated by the computer models described in Sect. IV. With these caveats, the density $\bar{\rho}$ is thus determined experimentally from a measurement of $\Delta p$, in accordance with (22).

A second determination of $\bar{\rho}$ results from the computer models themselves. This approach, however, relies not only on the validity of the transport modeling, but also on the specification of the initial flux of the beam as it leaves the nozzle of the source. Unfortunately, this specification contains substantial inherent uncertainties, as will become clear during the discus- sion of the Monte-Carlo ray tracing computations presented in Sect. IV B.

A third approach to the density determination involves the measurement of the angle integrated ion production rate, $I$, that results from electron impact ionization. In some sense this determination has the greatest relevance, since the polarized hydrogen beam line was designed specifically for studies of crossed beams electron-atom scattering. However, the density so obtained is an effective density, $\rho_{\text {eff }}$, specific to the geometry of the crossed beams interaction, which can be related to $\bar{\rho}$ only through detailed knowledge of the interaction volume and the variation of $\rho(r)$ within the volume. This point can be illustrated for a choice of coordinates in which the hydrogen beam lies along the $z$ axis and the electron beam lies along the $y$ axis, with both beams assumed to have cylindrical symmetry. In this case, the total number of atoms, $N^{\prime}$, in the interaction volume, $V$, can be expressed as

$N^{\prime}=\rho_{\text {eff }} V$

or more specifically as

$N^{\prime}=8 \int_{0}^{R_{e}} \mathrm{~d} z \int_{0}^{Y} \mathrm{~d} y \int_{0}^{X} \mathrm{~d} x \rho(r)$,

where $R_{e}$ is the effective radius of the electron beam, $r=\sqrt{x^{2}+y^{2}}$ is the radial coordinate applicable to the hydrogen beam, and the upper limits, $X$ and $Y$, are given by $X=\sqrt{R_{e}^{2}-z^{2}}$ and $Y=\sqrt{R_{H}^{2}-R_{e}^{2}+z^{2}}$. In terms of the electron current density, $j_{e}$ (expressed in electrons $\cdot \mathrm{cm}^{-2} \cdot \mathrm{s}^{-1}$ ), the ion production rate (expressed in ions $\cdot \mathrm{s}^{-1}$ ) can now be written as

$I=\varepsilon \sigma_{I} N^{\prime} j_{e}$,

where $\varepsilon$ is the overall experimental efficiency and $\sigma_{I}$ is the total ionization cross section.

From (23) and (25) it is clear that even if $\sigma_{I}$ is known, $\rho_{\text {eff }}$ can be obtained from measurements of $I$ and $j_{e}$, only if $V$ and $\varepsilon$ are known as well. The volume $V$, as illustrated by (24), depends not just upon $R_{H}$ but also on $R_{e}$, a dimension that unfortunately cannot be determined with great accuracy. The efficiency $\varepsilon$ also suffers from some uncertainty as will be seen in Sect. V. A comparison of (20), (23) and (24) shows moreover that the dependence of $\rho$ on $r$ must indeed be specified, if $\bar{\rho}$ is to be obtained from a measurement of $\rho_{\text {eff }}$.

All three methods of density determination are thus frought with deficiencies. Consequently, confidence in a quoted value of $\bar{\rho}$ requires reasonable consistency among the various results, an issue that will be addressed further in Sects. IV and V. 


\section{Dissociation fraction determination and $Q M A$ calibration}

The mass one and mass two signals produced by the QMA for an incident mixture of hydrogen atoms and hydrogen molecules can be written in terms of four response functions, $\alpha_{11}, \alpha_{12}, \alpha_{21}$, and $\alpha_{22}$. For incident densities $\rho_{1}^{q}$ and $\rho_{2}^{q}$, corresponding to atoms and molecules respectively, the specific relations for the mass one and mass two signals are given by

$Q_{1}=\alpha_{11} \rho_{1}^{q}+\alpha_{12} \rho_{2}^{q}$

and

$Q_{2}=\alpha_{21} \rho_{1}^{q}+\alpha_{22} \rho_{2}^{q}$,

where $Q_{1}$ and $Q_{2}$ in this section are understood to be beam related signals derived from chopper-open minus chopper-closed readings.

From the form of these equations, it is clear that $\alpha_{11}$ characterizes the mass one response of the QMA to atoms, while $\alpha_{22}$ characterizes the mass two response to molecules. The function $\alpha_{12}$, which produces a mass one response to incident molecules, results from dissociative ionization processes in the electron bombarder of the QMA. The function $\alpha_{21}$, which produces a mass two response to incident atoms, by contrast, results from recombination processes in the QMA and, for the geometry and atom densities involved, in fact may be neglected.

If the rf power at the hydrogen source is turned off, dissociation ceases, and the QMA signals become

$\left(Q_{1}\right)^{\text {off }}=\alpha_{12}\left(\rho_{2}^{q}\right)^{\text {off }}$

and

$\left(Q_{2}\right)^{\text {off }}=\alpha_{22}\left(\rho_{2}^{q}\right)^{\text {off }}$,

with the consequence that the ratio $\alpha_{12} / \alpha_{22}$ can be found from measurements of $\left(Q_{1}\right)^{\text {off }}$ and $\left(Q_{2}\right)^{\text {off }}$ according to the relation

$\left(\alpha_{12} / \alpha_{22}\right)=\left(Q_{1}\right)^{\text {off }} /\left(Q_{2}\right)^{\text {off }}$.

Note that (30) is applicable to any portion of the beam profile sampled by the QMA. If, however, measurements are restricted to the center of the profile, the dissociation fraction, $F^{0}$, at the source can be determined, where $F^{0}$ is defined as [36]

$F^{0} \equiv \frac{\left(\rho_{1}^{0}\right)^{\text {on }}}{\left(\rho_{1}^{0}\right)^{\text {on }}+\left(\rho_{2}^{0}\right)^{\text {on }}}$

for respective atom and molecule densities $\left(\rho_{1}^{0}\right)^{\text {on }}$ and $\left(\rho_{1}^{0}\right)^{\text {on }}$ at the exit of the source nozzle when the rf power is on. An expression for $F^{0}$ in terms of measurable QMA signals follows from the observation that when the rf power is turned on, the decrease in the $Q_{2}$ signal is directly attributable to the dissociation of each molecule into two atoms. Since the temperature of the source remains practically constant when the rf power is turned on, the molecular transport efficiency from the source to the QMA remains essentially unchanged. As a consequence, the ratio of densities $\left(\rho_{1}^{0}\right)^{\text {on }} /\left(\rho_{2}^{0}\right)^{\text {on }}$ for $\mathrm{rf}$ power on is given by

$$
\begin{aligned}
\frac{\left(\rho_{1}^{0}\right)^{\text {on }}}{\left(\rho_{2}^{0}\right)^{\text {on }}} & =2 \frac{\left(\rho_{2}^{0}\right)^{\text {off }}-\left(\rho_{2}^{0}\right)^{\text {on }}}{\left(\rho_{2}^{0}\right)^{\text {on }}} \\
& =2 \frac{\left(\rho_{2}^{q}\right)^{\text {off }}-\left(\rho_{2}^{q}\right)^{\text {on }}}{\left(\rho_{2}^{q}\right)^{\text {on }}}=2 \frac{\left(Q_{2}\right)^{\text {off }}-\left(Q_{2}\right)^{\text {on }}}{\left(Q_{2}\right)^{\text {on }}},
\end{aligned}
$$

where $\left(Q_{2}\right)^{\text {on }}$ and $\left(Q_{2}\right)^{\text {off }}$ are understood to be QMA readings in the central region of the beam profile. From (31) and (32) it is clear that $F^{0}$ can be written as

$F^{0}=\frac{2\left(R_{22}-1\right)}{2 R_{22}-1}$,

where the ratio $R_{22}$ is given by

$R_{22}=\left(Q_{2}\right)^{\text {off }} /\left(Q_{2}\right)^{\text {on }}$

Consider now a corresponding ratio

$r_{22}=\left(q_{2}\right)^{\text {off }} /\left(q_{2}\right)^{\text {on }}$

for QMA signals $\left(q_{2}\right)^{\text {on }}$ and $\left(q_{2}\right)^{\text {off }}$ obtained in the extreme wings, where, as in the case of signals taken from the central section, $\left(q_{2}\right)^{\text {on }}$ and $\left(q_{2}\right)^{\text {off }}$ are understood to be derived from chopper-open minus chopper-closed readings. Unlike those taken from the central section, however, the mass two signals obtained from the wings contain significant contributions from recombination of atoms that have struck the walls of the beam pipe in the vicinity of the QMA. Thus if $\beta_{1}$ and $\beta_{2}$ represent respectively the transport efficiencies from the source to the QMA for atoms and molecules, $\left(q_{2}\right)^{\text {on }}$ and $\left(q_{2}\right)^{\text {off }}$ can be written as

$\left(q_{2}\right)^{\text {on }}=\alpha_{22}\left[\frac{1}{2} \beta_{1}\left(\rho_{1}^{0}\right)^{\text {on }}+\beta_{2}\left(\rho_{2}^{0}\right)^{\text {on }}\right]$

and

$$
\begin{aligned}
\left(q_{2}\right)^{\text {off }} & =\alpha_{22} \beta_{2}\left(\rho_{2}^{0}\right)^{\text {off }} \\
& =\alpha_{22} \beta_{2}\left[\left(\rho_{2}^{0}\right)^{\text {on }}+\frac{1}{2}\left(\rho_{1}^{0}\right)^{\text {on }}\right],
\end{aligned}
$$

where the right side of (37) presumes no loss of atoms during the dissociation process. Through the combination of (32) and (34)-(37), the ratio of transport efficiencies $\beta_{1} / \beta_{2}$ can be expressed as 
$\frac{\beta_{1}}{\beta_{2}}=\frac{R_{22}-r_{22}}{r_{22}\left(R_{22}-1\right)}=\frac{\left(q_{2}\right)^{\text {on }}\left(Q_{2}\right)^{\text {off }}-\left(q_{2}\right)^{\text {off }}\left(Q_{2}\right)^{\text {on }}}{\left(q_{2}\right)^{\text {off }}\left[\left(Q_{2}\right)^{\text {off }}-\left(Q_{2}\right)^{\text {on }}\right]}$.

Finally with the ratio $\left(Q_{1}\right)^{\text {on }} /\left(Q_{2}\right)^{\text {on }}$ written as

$\frac{\left(Q_{1}\right)^{\mathrm{on}}}{\left(Q_{2}\right)^{\mathrm{on}}}=\frac{\alpha_{11} \beta_{1}\left(\rho_{1}^{0}\right)^{\mathrm{on}}+\alpha_{12} \beta_{2}\left(\rho_{2}^{0}\right)^{\mathrm{on}}}{\alpha_{22} \beta_{2}\left(\rho_{2}^{0}\right)^{\mathrm{on}}}$,

it can be shown with the assistance of (30), (34), (35) and (38) that the QMA response functions $\alpha_{11}$ and $\alpha_{22}$ are related by

$\frac{\alpha_{11}}{\alpha_{22}}=\frac{1}{2} \frac{\left(q_{2}\right)^{\text {off }}\left(Q_{1}\right)^{\text {on }}-\left(q_{1}\right)^{\text {off }}\left(Q_{2}\right)^{\text {on }}}{\left(q_{2}\right)^{\text {on }}\left(Q_{2}\right)^{\text {off }}-\left(q_{2}\right)^{\text {off }}\left(Q_{2}\right)^{\text {on }}}$.

Thus, (30) and (40) provide access to the relative calibration of the QMA for masses one and two, while (38) provides access to the relative transport efficiencies from the source to the QMA for atoms and molecules. Finally, (33) and (34) provide the necessary relations for the dissociation fraction $F^{0}$ at the source to be determined.

\section{Computer models}

\section{A. General consideration}

As suggested in Sect. III the determination of hydrogen beam density, polarization, and dissociation fraction cannot easily be made by experimental measurement alone. This is particularly true if the beam parameters need to be known at the interaction region, a location at which the requisite measurement devices cannot be placed without causing severe disruption of the beam line itself. Thus, detailed theoretical modeling of the beam becomes an integral part of the measurement process, placing great importance on the confidence with which the calculational results are held.

In order to increase the reliability of our results, we therefore used three different modeling methods: (1) a Monte-Carlo ray-tracing analysis, (2) an approach that treated the hexapole state selector as a thick lens, and (3) a Cartesian phase space calculation. All three techniques relied on the application of standard kinetic gas theory to establish the operating density of the source in terms of the ballast volume pressure. In the following sections we will review the principal elements of the calculations and present summaries of their predictions.

\section{B. Source density and gas kinetics}

All three calculational methods require specification of the hydrogen density either inside the source or

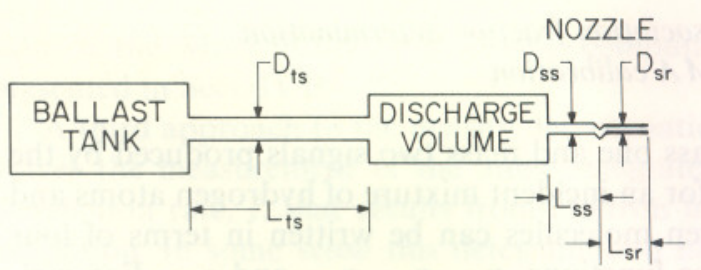

Fig. 11. Schematic diagram of the hydrogen gas transport system showing ballast tank, connecting tubing, and rf discharge volume with nozzle

immediately outside the discharge nozzle. Since direct measurement of the discharge pressure is precluded by the design of the source, the kinetic theory of gases must be used to extract the information from pressure measurements carried out at the hydrogen ballast tank [21, 35, 37]. Unfortunately, the conditions under which the source operates makes this extraction very difficult and leads to some uncertainty in the effective operating pressure.

The nature of the difficulty is best illustrated with the aid of the schematic diagram shown in Fig. 11, which depicts the ballast tank, the discharge volume, and the discharge nozzle, as well as the connections between the elements. The connection between the ballast tank and the discharge volume can be reasonably characterized by an effective diameter $D_{t s} \approx 6 \mathrm{~mm}$ and an effective length $L_{t s} \approx 800 \mathrm{~mm}$. The nozzle, however, is more complex, having an entry section, with a diameter $D_{s s}=3 \mathrm{~mm}$ and a length $L_{s s}=25 \mathrm{~mm}$, and an exit section, with a diameter $D_{s r}=1 \mathrm{~mm}$ and a length $L_{s r}=25 \mathrm{~mm}$. Separating the two sections is a kink that reduces the Lyman- $\alpha$ emission of the source, and it is this kink combined with the operating pressure of the source that creates the major difficulty.

At the operating ballast tank pressure $p_{t} \approx 400$ mTorr, the mean free path of molecular hydrogen, $\lambda_{\mathrm{H}_{2}}$, is approximately $0.2 \mathrm{~mm}$, which places the molecular gas transport from the ballast tank to the discharge volume in the intermediate flow regime [35]. The conductance of the transport tube, $C$, must then be expressed in the form

$C=\left(c_{1} D^{4} \bar{p}+c_{2} D^{3}\right) / L$,

where $\bar{p}$ is the average pressure in the tube; where the parameter $c_{1}$ depends simply upon the viscosity of the gas, $\eta$; where the parameter $c_{2}$ depends in a complex fashion upon the molecular weight, $M$, and the Kelvin temperature, $T$, of the gas in the combination $\sqrt{T M}$, as well as upon the ratio $D \bar{p} / \eta$; and where the subscripts on $C, D, L$, and $\bar{p}$ are implicit. The limiting condition of molecular flow occurs when the pressure and temperature of the gas are such that the mean free path $\lambda$ satisfies the inequality $\lambda / D>1$. In this case, the term $c_{2} D^{3}$ dominates in (41). On 
the other hand, when the condition $\lambda / D<0.01$ obtains, the limiting case of viscous flow is achieved, and it is the term $c_{1} D^{4} \bar{p}$ that dominates in (41).

Although the intermediate flow regime is somewhat complicated to handle, it is nonetheless amenable to analytic calculation. It applies along the entire length of the transport tube between the ballast tank and the discharge volume, since the pressure gradient along the tube is quite small, and $\lambda_{\mathrm{H}_{2}}$ consequently remains close to $0.2 \mathrm{~mm}$ throughout. The situation in the nozzle, by contrast, is far more complex, since not only must the intermediate flow regime be anticipated, but also some turbulence must be assumed because of the presence of the kink.

Regardless of the details, however, it is clear from the relative dimensions of the nozzle that the conductance of the nozzle, $C_{s r}$, is considerably smaller than that of the transport tube, $C_{t s}$. Moreover, the difference is only enhanced by the presence of turbulence in the nozzle. Under these circumstances, the pressure $p_{s}$ in the discharge tube can be estimated from the throughput relations

$\mathscr{Q}_{t s}=C_{t s}\left(p_{t}-p_{s}\right)$

and

$\mathscr{Q}_{s r}=C_{s r}\left(p_{s}-p_{r}\right)$,

where $\mathscr{2}_{t s}$ and $\mathscr{Q}_{s r}$ are the throughputs in the transport tube and the nozzle respectively, and $p_{r}$ is the vacuum chamber residual gas pressure. If the dissociation fraction $F^{0}$ is taken to be approximately 0.85 , consistent with measurement, it follows that $\mathscr{Q}_{s r} \approx 1.75 \mathscr{2}_{t s}$, in which case (42) and (43) lead to the expression

$p_{s} \approx\left[\frac{4}{7} \frac{C_{s r}}{C_{t s}}+1\right]^{-1} p_{t}$

where use has been made of the relation $p_{s} \gg p_{r}$. Bounds on the ratio $C_{s r} / C_{t s}$ can be found from the limiting cases of purely viscous flow and purely molecular flow, with turbulence neglected. For these two cases the conductance given by (41) can be written as [35]

$\left.C=3.27 \times 10^{-2}\left[D^{4} / \eta L\right)\right] \bar{p} \quad$ Viscous

and

$C=3.81(T / M)^{\frac{1}{2}}\left(D^{3} / L\right), \quad$ Molecular

where dimensions are expressed in $\mathrm{cm} ; \eta$, in poise; $\bar{p}$, in Torr; and $C$, in $l / s$. It is clear that (46) leads to the upper bound on the ratio $C_{s r} / C_{t s}$, and with appropriate values used for $M, D$, and $L$ for the nozzle and the transport tube it follows that $C_{s r} / C_{t s}<0.2$, assuming constant $T$ throughout the system. Applica- tion of (44) immediately leads to the condition $p_{t}$ $>p_{s}>0.9 p_{t}$. For purposes of further estimation, we therefore use the approximate value of $380 \mathrm{mT}$ Torr for $p_{s}$.

In order to model the flow out of the nozzle, we first observe that at a pressure of $380 \mathrm{mTorr}$, the mean free path of atomic hydrogen, $\lambda_{H}$, is of the order of $0.5 \mathrm{~mm}$. Thus, effusive flow conditions do not apply along the full length of the nozzle, and the usual expression for the number of atoms leaving the nozzle

$\mathscr{I}^{0}=\frac{\rho_{1}^{s}\langle v\rangle \pi R_{s r}^{2}}{4 \kappa}$

does not apply [37], where $\langle v\rangle$ is the mean velocity of atoms in the source [21], $R_{s}$ is the effective radius of the nozzle and $\kappa$ is given by

$\kappa=\frac{3}{8} \frac{L_{s r}}{R_{s r}}$,

with $L_{s r}$ the effective length of the nozzle. A more reasonable characterization of the nozzle, perhaps, relies on an assumption of intermediate flow up to the kink, a turbulent condition at the kink with a consequent small $(\sim 10 \%)$ reduction of pressure, and finally an approach to effusive flow very close to the nozzle exit. Given the uncertainties in the model, we simply assume an approximate hydrogen gas pressure $p_{s s}$ of 350 mTorr near the nozzle exit, and with $\lambda_{H} \sim R_{s r}$ we accept the effusive flow relation for beam formation at an aperture; namely $[21,37]$

$\mathscr{I}^{0}=\frac{\rho_{1}^{s s}\langle v\rangle \pi R_{s r}^{2}}{4}$.

We further assume the normal $\cos \theta$ distribution associated with effusive flow. We note finally that in terms of $\mathscr{I}^{0}$, the density of atoms just outside the nozzle, $\rho_{1}^{0}$, can be written as

$\rho_{1}^{0}=\frac{\mathscr{I}^{0}}{\langle v\rangle \pi R_{s r}^{2}}$

as a consequence of which $\rho_{1}^{0}$ is given by

$\rho_{1}^{0}=\rho_{1}^{s s} / 4$.

For an assumed pressure $p_{s s} \sim 350 \mathrm{mTorr}$, the ideal gas law leads to a density $\rho_{1}^{s s} \sim 10^{16}$ atoms $/ \mathrm{cm}^{3}$ just before the nozzle exit and consequently to a density $\rho_{1}^{0} \sim 2.5 \times 10^{15}$ atoms $/ \mathrm{cm}^{3}$ just outside the nozzle.

\section{Monte-Carlo ray tracing}

Using the value of $\rho_{1}^{0}$ for normalization and assuming the $\cos \theta$ distribution characteristic of an effusive flow 


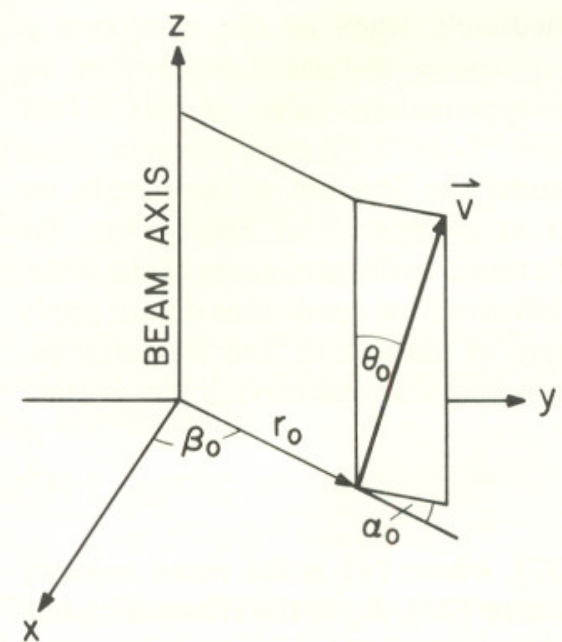

Fig. 12. Coordinate system and angles used in Monte-Carlo ray tracing computation

source, we carried out a Monte-Carlo analysis of the hydrogen beam. In terms of the coordinate system and angles shown in Fig. 12, we specified initial conditions for each trajectory as follows: $\alpha_{0}, \beta_{0}, \theta_{0}, r_{0}$, and $v_{0}$ where the initial velocity $\mathbf{v}_{0}$, is given by

$$
\begin{aligned}
\mathbf{v}_{0}= & v_{0}\left\{\sin \theta_{0}\left[\cos \left(\alpha_{0}+\beta_{0}\right) \hat{x}+\sin \left(\alpha_{0}+\beta_{0}\right) \hat{y}\right]\right. \\
& \left.+\cos \theta_{0} \hat{z}\right\},
\end{aligned}
$$

and the initial radial coordinate $r_{0}$ is related to the initial cartesian coordinates by the usual expressions $x_{0}=r_{0} \cos \beta_{0}$ and $y_{0}=r_{0} \sin \beta_{0}$. Employing the subscript zero to denote the initial value of the relevant quantity at the start of a computer step, we can summarize the equations of motion as follows:

(1) In drift regions,

$$
\begin{aligned}
& x=x_{0}+\left.\left(z-z_{0}\right) \frac{\mathrm{d} x}{\mathrm{~d} z}\right|_{0} \\
& y=y_{0}+\left.\left(z-z_{0}\right) \frac{\mathrm{d} y}{\mathrm{~d} z}\right|_{0} .
\end{aligned}
$$

(2) Inside the hexapole magnet [30], with $M_{H}$ the mass of the hydrogen atom, $v \approx v_{z}$ the velocity of the hydrogen atom, and $K$ defined by

$$
\begin{aligned}
K= & \frac{1}{v}\left[\frac{\mu_{\mathrm{eff}} H\left(R_{M}\right)}{M_{H} R_{M}^{2}}\right]^{\frac{1}{2}}, \\
& (a) \text { for } \mu_{\mathrm{eff}}<0 \\
x= & x_{0} \cos (K z)+\left.\frac{1}{K} \frac{\mathrm{d} x}{\mathrm{~d} z}\right|_{0} \sin (K z) \\
y= & y_{0} \cos (K z)+\left.\frac{1}{K} \frac{\mathrm{d} y}{\mathrm{~d} z}\right|_{0} \sin (K z) ;
\end{aligned}
$$

(b) for $\mu_{\text {eff }}>0$

$x=x_{0} \cosh (K z)+\left.\frac{1}{K} \frac{\mathrm{d} x}{\mathrm{~d} z}\right|_{0} \sinh (K z)$

$y=y_{0} \cosh (K z)+\left.\frac{1}{K} \frac{\mathrm{d} y}{\mathrm{~d} z}\right|_{0} \sinh (K z)$.

(3) Inside the Stern-Gerlach magnet [21] with $\mathfrak{U}$ the radius of the convex pole piece and with the field and its gradient given respectively by

$H=\frac{2 \mathfrak{U}^{2}}{\mathfrak{U}^{2}+x^{2}} H(\mathfrak{U})$

and

$\frac{\partial H}{\partial x}=-\frac{4 \mathfrak{U}^{2} x}{\left(\mathfrak{Q}^{2}+x^{2}\right)^{2}} H(\mathfrak{Q})$,

$x=x_{0}+\left.\left(z-z_{0}\right) \frac{\mathrm{d} x}{\mathrm{~d} z}\right|_{0}+\frac{1}{2} \frac{\mu_{\mathrm{eff}}}{M_{H}} \frac{\partial H}{\partial x}\left[\frac{z-z_{0}}{v}\right]^{2}$

$y=y_{0}+\left.\left(z-z_{0}\right) \frac{\mathrm{d} y}{\mathrm{~d} z}\right|_{0}$.

For many trajectories, the magnitude of the field inside the hexapole and Stern-Gerlach magnets remains sufficiently high that within these regions $\mu_{\text {eff }}$ remains constant and equal to $\pm \mu_{B}$. As a consequence, for these trajectories the computer step size is irrelevant, and (56)-(59), (62), and (63) may be regarded as analytic equations, with the subscript zero denoting the value of the relevant quantity at the entrance to the particular region. For other trajectories, however, the step size is critical and must be examined with great care. For all trajectories, the drift region behavior described by (53) and (54), clearly satisfies the requirement for analytic treatment.

Regardless of circumstance, the characteristics of the beam at any location, $z$, along the beam line requires a tally of the trajectories arriving at that point. To that end, we set up bins of dimension $\Delta x$ and $\Delta y$ and calculated the density $\rho_{1}^{z}(x, y)$, the low-field polarization $P^{z}(0 ; x, y)$, and the high-field state-selection parameter $s^{z}(x, y)$ according to the respective prescriptions

$\rho_{1}^{z}(x, y)=\frac{1}{n_{\mathrm{tot}}} \frac{n^{z}(x, y)}{\Delta x \Delta y} \pi R_{s r}^{2} \rho_{1}^{0}$,

$P^{z}(0 ; x, y)=\frac{n_{++}^{z}(x, y)-n_{--}^{z}(x, y)}{n^{z}(x, y)}$,

and 

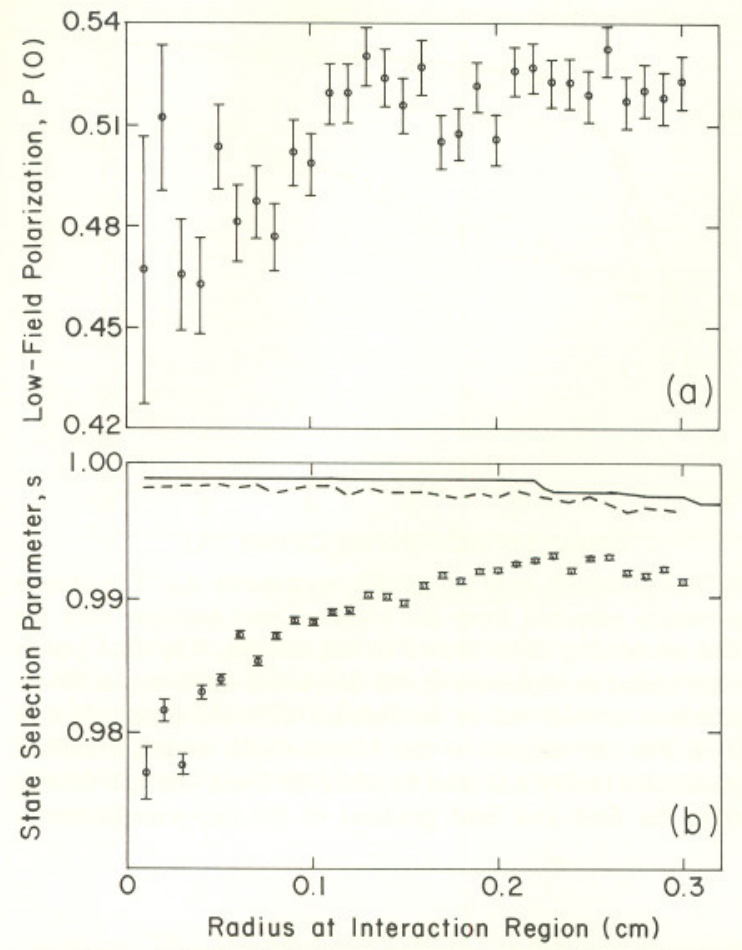

Fig. 13a and b. Radial dependence of low-field polarization $P(0)$ in (a) and high-field state selection parameter $s$ in (b) at the interaction region. Points with error bars reflecting counting statistics were obtained from the field-dependent $\mu_{\text {eff }}$ Monte-Carlo computation, while the solid and dashed lines represent the results of the optical model and Monte-Carlo analysis respectively for constant $\mu_{\text {eff }}$ $= \pm \mu_{B}$

$s^{z}(x, y)$

$=\frac{\left(n_{++}^{z}(x, y)+n_{+-}^{z}(x, y)\right)-\left(n_{-+}^{z}(x, y)+n_{--}^{z}(x, y)\right)}{n^{z}(x, y)}$,

where $n_{\text {tot }}$ is the total number of trajectories starting from the source, $n^{z}(x, y)$ is the total bin tally, and $n_{++}^{z}(x, y)$ is the bin tally corresponding to the highfield spin assignments $\left(m_{S}, m_{I}\right)=\left(+\frac{1}{2},+\frac{1}{2}\right)$, with the remaining $n$ 's correspondingly defined.

The results of the Monte-Carlo computations, based upon a sample of $10^{10}$ atoms effusing from the source and reduced by the conservative solid angle acceptance of downstream beam elements to 4.6 $\times 10^{6}$ interrogated trajectories, are summarized in Figs. 13-18 which illustrate the beam characteristics at the interaction region, at the entrance to the SternGerlach magnet, and at the entrance to the QMA, the last two of which assume the insertion of all collimating slits. It should be noted that in obtaining these results we treated both magnets as ideal systems with all fringe fields completely neglected. As can be seen from Fig. 13, the low field polarization, $P(0)$, and the high-field state selection parameter $s$, vary only slightly over the volume of the interaction region, the
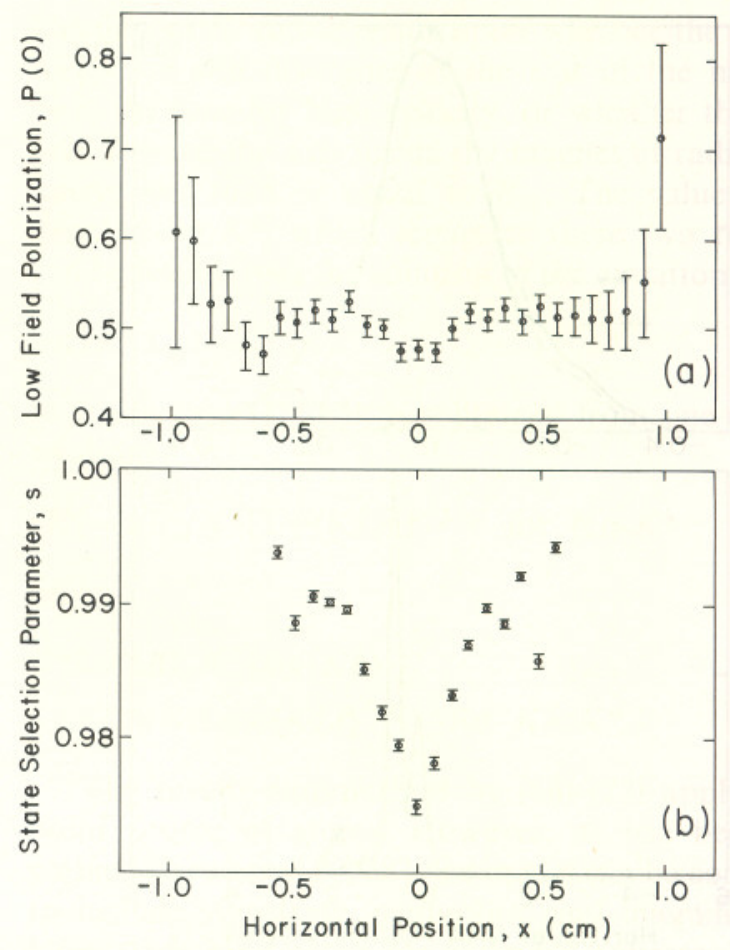

Fig. 14a and b. Results of field dependent $\mu_{\text {eff }}$ Monte-Carlo analysis for the low-field polarization $P(0)$ in (a) and high-field state selection parameter $s$ in (b) as functions of the horizontal position of the first acceptance slit of the Stern-Gerlach polarimeter. The data represent convolutions over the acceptance dimensions of the polarimeter ( $0.7 \mathrm{~mm}$ wide by $2.5 \mathrm{~mm}$ high) with the error bars reflecting counting statistics only

former remaining close to 0.5 and the latter, close to unity throughout. With the uncertainty reflecting counting statistics only, the average value $\bar{P}(0)$ of the low-field polarization at the interaction region is $0.515(2)$, while the average value of the state selection parameter $\bar{s}$ is $0.99102(4)$. It should be noted that if $\mu$ is improperly treated as a constant $\pm \mu_{B}$, the radial dependence of $s$ changes its character, as shown in Fig. 13(b), and the value of $\bar{s}$ becomes $0.99758(3)$. With the application of (14) and (16), $P(0)$ becomes $0.49879(2)$. The discrepancy, while small for $\bar{s}$ is larger for $P(0)$ and should be of more than passing interest to other groups working with hexapole state selectors.

Figure 14 illustrates the variation of $P(0)$ and $s$ with the horizontal position of the first entrance slit of the Stern-Gerlach polarimeter. Representing convolutions over the $0.7 \mathrm{~mm}$-wide by $2.5 \mathrm{~mm}$-high acceptance dimensions of the polarimeter, the data points in Fig. 14 continue to reflect (with some enhancement) the same small decrease on axis for both $P(0)$ and $s$ that is visible in Fig. 13. The presence of the axial dips plays a significant role in the interpretation of the polarization measurements described in Sect. V. 

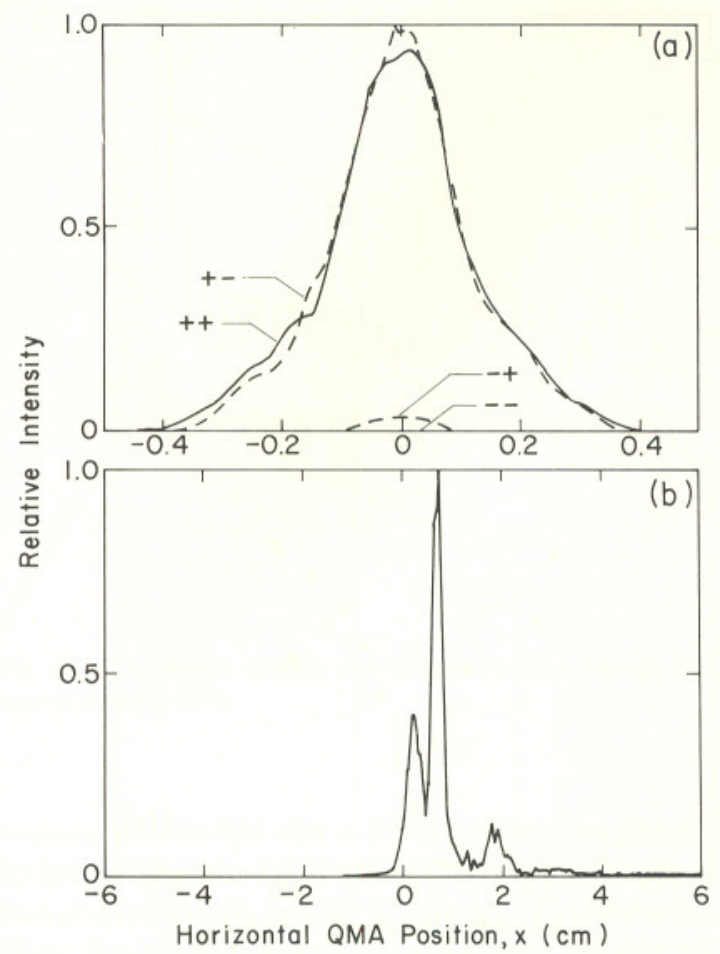

Fig. 15a and b. Monte-Carlo generated beam profiles containing convolutions with the horizontal acceptance window of the QMA. The results in (a), obtained with the Stern-Gerlach magnet turned off, are shown separately for the four hyperfine magnetic substates and are labeled in accordance with the high-field designations $\left(m_{S}, m_{I}\right)$. The profile in (b) is the combined result for all substates obtained with a $5 \mathrm{~A}$ driving current in the Stern-Gerlach magnet

For the purpose of relating the actual measurements carried out with the Stern-Gerlach polarimeter to the low-field beam polarization present in the interaction region, we propagated the trajectories downstream through the polarimeter and into the QMA, convoluting the binned results with a horizontal window $0.5 \mathrm{~mm}$ wide which simulates the true acceptance slit of the mass analyzer. Figure 15(a) illustrates the Monte-Carlo beam profile obtained when the SternGerlach magnet is deenergized and when its collimating aperture is centered on the beam axis. For completeness we have shown separately the four hyperfine magnetic sublevels labeled in accordance with their $\left(m_{S}, m_{I}\right)$ high-field designations. As the energizing current increases from zero, the ++ and +- profiles shift toward the right and the -- and -+ profiles shift toward the left, so that at a current of $5 \mathrm{~A}$, the combined profile takes on the appearance shown in Fig. 15(b). The peaked structures appearing in the profile are real and are related to the velocity classes that are preferentially transmitted by the hexapole magnet.

For each of fifteen settings of the Stern-Gerlach driving current between 0 and $6 \mathrm{~A}$, we divided the

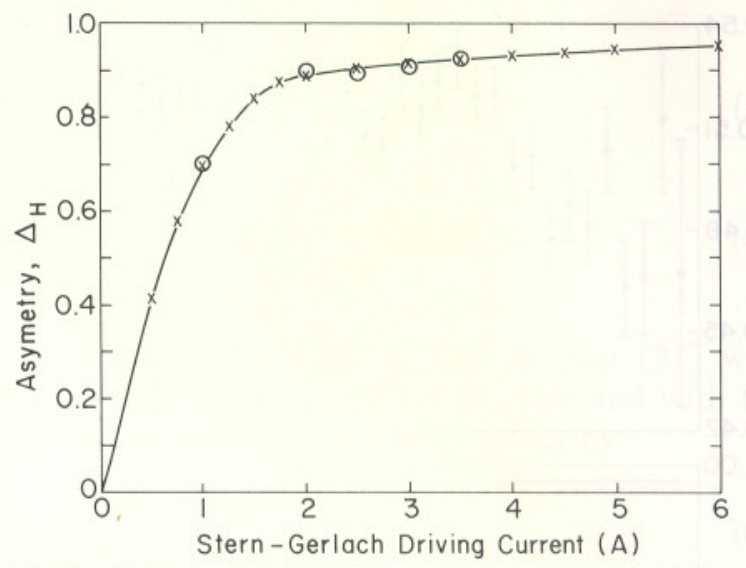

Fig. 16. Stern-Gerlach right-left profile asymmetry $\Delta_{H}$. The crosses are the results obtained from the Monte-Carlo analysis, with the solid line connecting them drawn to aid the eye. The data points shown are scaled as explained in the text based upon actual QMA measurements carried out in accordance with the description in Sect. III B. For the display of the Monte-Carlo results, standard magnetic circuit theory was used to relate the Stern-Gerlach driving current to the field and field gradient of the two-wire geometry [15]

profile area into regions to the right, $A_{R}$, and to the left, $A_{L}$, of the beam axis, and, by analogy with the procedures described in Sect. III.B for the reduction of the laboratory data, we formed the asymmetry $\Delta_{H}$ according to the prescription

$\Delta_{H}=\frac{A_{R}-A_{L}}{A_{R}+A_{L}}$.

The crosses and the curve connecting them in Fig. 16 illustrate the results for the Monte Carlo asymmetry so generated. The high-field state selection parameter $s$, obtained from the Monte-Carlo analysis when the Stern-Gerlach polarimeter is centered on the beam axis, is given by the asymptotic limit of $\Delta_{H}$, which has the value $0.9821(4)$.

As the last illustration of the output of the MonteCarlo computation, we present in Fig. 17 plots of the radial dependence of the total atom density at the interaction region for two cases: a field-dependent $\mu_{\text {eff }}$ and a constant $\mu_{\mathrm{eff}}= \pm \mu_{B}$. The plots are truncated at a radius of $3 \mathrm{~mm}$ in accordance with the cutoff imposed by the collimating aperture located $38 \mathrm{~mm}$ upstream from the crossed-beams interaction center. The Monte-Carlo computation for the field dependent $\mu_{\text {eff }}$ case predicts an average density $\bar{\rho}$ of $9 \times 10^{9}$ atoms $/ \mathrm{cm}^{3}$ over the approximately $7 \mathrm{~mm}$ diameter beam present at the interaction region.

\section{Optical model of hexapole magnet}

With the use of (55)-(59) it is possible to develop a model of the hexapole magnet in terms of an optical 


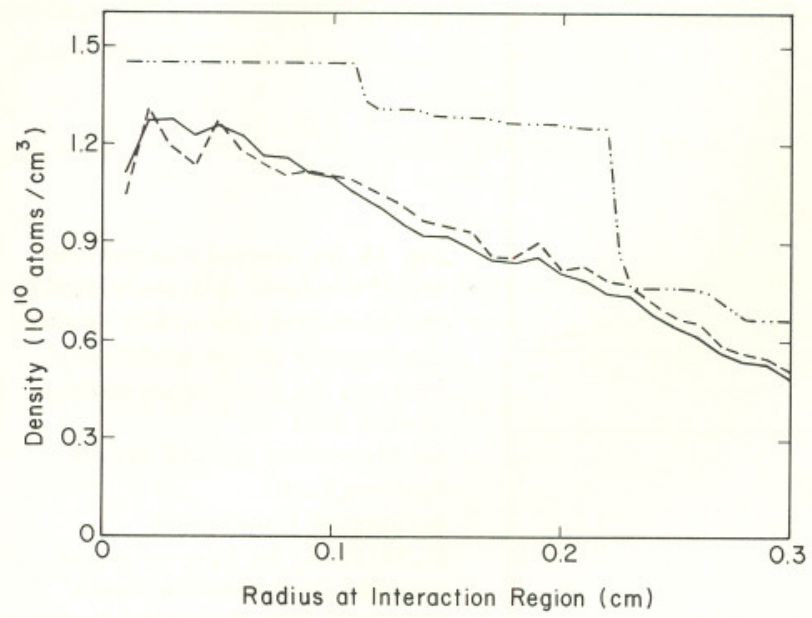

Fig. 17. Atomic density $\rho$ at the interaction region as a function of radial position. The solid and dashed lines are the results from the Monte-Carlo analysis for the field-dependent $\mu_{\text {eff }}$ and the constant $\mu_{\text {eff }}= \pm \mu_{B}$ cases, respectively. Also shown is the result from the optical model (dashed line with dots) which assumes a constant $\mu_{\text {eff }}= \pm \mu_{B}$

analog. This approach, which has been described in detail elsewhere [30,38], assumes the high-field approximation for $\mu_{\mathrm{eff}}$ and determines the density and state-selection parameter of the beam from the spectral transmittance $\Omega_{ \pm}(K)$ of the magnet for atoms in the $m_{S}= \pm \frac{1}{2}$ state and the $v^{2}$-Maxwellian distribution function $F_{v^{2}}(K)$ expressed in terms of inverse velocity parameter defined by (55). The number of atoms reaching the interaction region within a radius $R_{H}$ located a distance $L_{2}$ from the exit of the magnet is then given by

$N_{ \pm}=$(const) $\int_{0}^{\infty} F_{v_{2}}(K) \Omega^{ \pm}\left(K, L_{1}, L_{2}, L_{M}, R_{H}\right) \mathrm{d} K$,

where $L_{1}$ is the distance from the source to the entrance of the magnet, and $L_{M}$ is the length of the magnet.

For atoms in the $m_{S}=-\frac{1}{2}$ state, $\Omega_{-}$can be shown to be given by

$$
\begin{aligned}
\Omega_{-}= & \pi R_{H}^{2}\left[\left(L_{1} L_{2} K+1 / K\right) \sinh \left(K L_{M}\right)\right. \\
& \left.+\left(L_{1}+L_{2}\right) \cosh (K L)\right]^{-2} .
\end{aligned}
$$

Similarly, for atoms in the $m_{S}=+\frac{1}{2}$ state, $\Omega_{+}$can be shown to be given by

$$
\begin{aligned}
\Omega_{+}= & \pi R_{H}^{2}\left[\left(L_{1} L_{2} K-1 / K\right) \sin (K L)\right. \\
& \left.-\left(L_{1}+L_{2}\right) \cos (K L)\right]^{-2},
\end{aligned}
$$

provided the result does not exceed the acceptance angle $\Omega_{+}^{\text {acc }}$ of the magnet itself. The latter is also a function of $K$ and depends upon whether the trajectories are still diverging at the exit of the magnet, as is the case for high velocity, or whether they are bent toward the axis inside the magnet at radial distances less than or equal to $R_{M}$. The value of $K$, denoted by $K^{*}$, which separates these two regimes can be found from the solution of the equation

$\cot \left(K^{*} L_{M}\right)=K^{*} L_{1}$,

in which case $\Omega_{+}^{\text {acc }}$ can be obtained from one of two relations:

$\Omega_{+}^{\mathrm{acc}}=\pi R_{M}^{2} K^{2}\left[1+(K L)^{2}\right]^{-1}$ for $K>K^{*}$

or

$$
\begin{aligned}
\Omega_{+}^{\mathrm{acc}}= & \pi R_{M}^{2} K^{2}[\sin (K L) \\
& +K A \cos (K L)]^{-2} \text { for } K<K^{*} .
\end{aligned}
$$

The results summarized by (68)-(73) apply to a point source of atoms. However, as has been explained elsewhere $[30,38]$, the model can be corrected to include vignetting, penumbra, and magnification losses that may occur for extended sources. In the case of the hydrogen beam under discussion, for which the source diameter is small compared to the magnet pole-gap, the corrections are small and amount to changes of no more than a few percent of the values calculated.

We employed the optical model of the hexapole magnet, including the effects of vignetting, penumbra and magnification, to obtain values of $\Omega_{ \pm}$in accordance with (69)-(73), and used these results together with (15) and (68) to obtain the state selection parameter $s$, as a function of radius at the interaction region, as shown in Fig. 13(b). The results are in reasonable agreement with those already presented in Fig. 13(b) for the Monte-Carlo computations carried out with constant $\pm \mu_{\boldsymbol{B}}$. Moreover the value of 0.998 obtained for $\bar{s}$ from the optical model agrees well with the value of 0.99758 (3) obtained from the constant $\pm \mu_{B}$ MonteCarlo analysis.

The results of the optical model combined with the relations

$\Omega=\int_{0}^{\infty} F_{v^{2}}(K) \frac{1}{2}\left[\Omega_{+}(K)+\Omega_{-}(K)\right] \mathrm{d} K$

and

$\bar{\rho}\left(L_{1}, L_{2}, L_{M}, R_{H}\right) \approx \frac{\rho_{1}^{s s} \Omega R_{s r}^{2}}{4 R_{H}^{2}}$,

lead us to a value of $1 \times 10^{10}$ atoms $/ \mathrm{cm}^{3}$ for the average density of atoms in the interaction region, which is also in reasonable agreement with the value ob- 

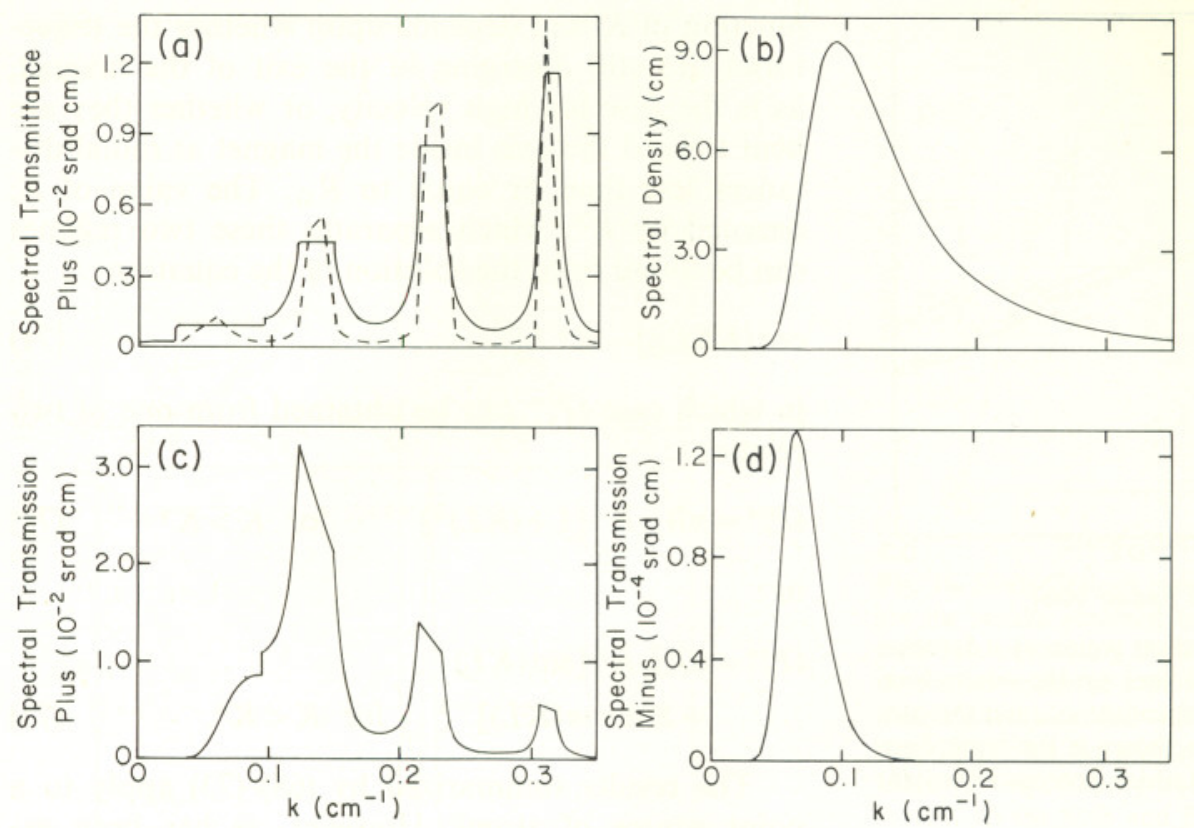

Fig. 18. (a) Spectral transmittance $\Omega_{+}(K)$ for $m_{S}=+\frac{1}{2}$ atoms reaching the interaction region. The results are from the optical model (solid line) and the phase space method (dashed line).

(b) Maxwellian spectral density function $F_{v^{2}}(K)$.

(c) Spectral transmission $\Omega_{+}(R) F_{v^{2}}(K)$ for $m_{S}=+\frac{1}{2}$ atoms reaching the interaction region, calculated from the optical model. (d) Spectral transmission $\Omega_{-}(K) F_{v^{2}}(K)$ for $m_{S}=-\frac{1}{2}$ atoms reaching the interaction region, calculated from the optical model tained from the Monte-Carlo analysis. However, the dependence of the density on the radial position at the interaction region shows some small disagreement between the two methods, as illustrated by a comparison of the curves in Fig. 17. For completeness, it should be noted that in obtaining the results from the optical model we treated the pair of hexapole magnets as a single unit $323 \mathrm{~mm}$ long without any gap between them. In addition we treated them as ideal hexapoles without any other moments being represented and with fringe fields neglected, as was the case for the Monte-Carlo calculations described in the previous section.

As was suggested in the previous section, the chromatic properties of the hexapole magnet lead to the assignment of a common focal plane for a series of velocity classes of $m_{S}=+\frac{1}{2}$ atoms. This point is well illustrated by Fig. 18(a), in which the spectral transmittance function $\Omega_{+}(K)$ is plotted versus $K$ for atoms reaching the interaction region. With the use of the $v^{2}$-Maxwellian distribution function, $F_{v^{2}}(K)$, shown in Fig. 18(b), the corresponding spectral transmission function $\Omega_{+}(K) F_{v^{2}}(K)$ can be obtained, as depicted in Fig. 18(c). The three peaks visible in Fig. 18(c) correspond to the three peaks in the beam profile of Fig. 15(b). From a comparison of Fig. 18(b) and (c) it is apparent that the average value $\bar{K}$ associated with the spectral transmission is shifted upward from the average value $\langle K\rangle$ associated with $F_{v^{2}}(K)$ alone. Hence, as we stated in Sect. IIIC, the average value $\bar{v}$ that characterizes the beam at the interaction region is lower than that ordinarily associated with the $v^{2}$-Maxwelliam appropriate to density calculations; namely, $\langle v\rangle=\sqrt{\frac{8 k T}{\pi M_{H}}}$. For completeness, we have included a plot of the spectral transmission $\Omega_{-}(K) F_{v^{2}}(K)$, shown in Fig. 18(d).

\section{E. Cartesian phase space calculation}

The phase space method has been successfully employed $[21,39]$ a number of times to calculate intensities and polarizations of state-selected and focused beams of atoms and molecules. In fact, the technique has been specifically applied to the case of magnetically state-selected hydrogen in a situation not too different from ours [21]. The parameterization of the problem derives from the harmonic equation of an atom in a hexapole magnet which leads to (56)-(59). Specifically, in any direction, $x$, perpendicular to the beam direction $z$, the equation takes the form

$\frac{\mathrm{d}^{2} x}{\mathrm{~d} t^{2}}+\omega^{2} x=0$,

where $\omega$ is given by

$\omega=v K=\left[\frac{\mu_{\text {eff }} H_{R}}{M_{H} R_{M}^{2}}\right]^{\frac{1}{2}}$.

With $p_{x}=M_{H} v_{x},(76)$ can be recast as

$$
\frac{p_{x}^{2}}{2 M_{h}}+\frac{M_{H} \omega^{2} x^{2}}{2}=W
$$


where $W$ is a constant. Using the paraxial approximation, we can express $p_{x}$ as

$p_{x}=M_{H} \frac{\mathrm{d} x}{\mathrm{~d} t}=M_{H} \frac{\mathrm{d} x}{\mathrm{~d} z} \frac{\mathrm{d} z}{\mathrm{~d} t}=M_{H} v_{z} \tan \phi_{x} \approx M_{H} v \phi_{x}$,

where $\phi_{x}$ is the angle that the tangent to the trajectory makes with the $z$ axis. Then with $\xi_{x}$ and $\eta_{x}$ defined respectively by $\xi_{x} x / R_{M}$ and $\eta_{x} \phi_{x} / K R_{M}$, (78) can be rewritten in dimensionless form as

$\xi_{x}^{2}+\eta_{x}^{2}=w$,

where $w$ is given by

$w=\frac{2 W}{M_{H} \omega^{2} R_{M}^{2}}$.

Thus in the phase space described by $\left(\xi_{x}, \eta_{x}\right)$, the trajectories inside a hexapole magnet are circles of radius $\sqrt{w}$ centered at the origin. For a magnet of length $L_{M}$, the effect on an ensemble of atoms in $\left(\xi_{x}, \eta_{x}\right)$ space is a rotation of all points counterclockwise about the origin through an angle $K L_{M}$.

In a field free region, the equations of motion for $\chi$ and $\phi_{x}$ have the obvious forms $\phi_{x}=\left(\phi_{x}\right)_{0}$ and $x$ $=x_{0}+\phi_{x} z$, where the subscript zero denotes the initial value of each parameter. Thus in terms of $\xi_{x}$ and $\eta_{x}$, all trajectories satisfy the relations

$\eta_{x}=\left(\eta_{x}\right)_{0}$

and

$\xi_{x}=\left(\xi_{x}\right)_{0}+K z \eta_{x}$

where $\left(\eta_{x}\right)_{0}$ and $\left(\xi_{x}\right)_{0}$ are constants related to $\left(\phi_{x}\right)_{0}$ and $x_{0}$ respectively through the definitions of $\xi_{x}$ and $\eta_{x}$. Therefore the effect on an ensemble of atoms undergoing field-free motion is a shearing action in $\left(\xi_{x}, \eta_{x}\right)$ space, with points above the horizontal $\xi_{x}$ axis displaced to the right and those below the $\xi_{x}$ axis displaced to the left, all displacements being proportional to the distance of the points from the $\xi_{x}$ axis.

Following the application of Liouville's theorem and the phase space arguments presented elsewhere, it can be shown that the total number of $m_{S}= \pm \frac{1}{2}$ atoms per second passing through a region of interest of area $\Sigma$ along the beam line downstream from the hexapole magnet as given by

$\mathscr{I}_{ \pm}^{\Sigma}=\frac{1}{4 \pi} \frac{\rho_{1}^{s s}}{2}\langle v\rangle R_{M}^{4} \int_{0}^{\infty}\left[A^{\Sigma}(K)\right]^{2} K^{2} F_{v}(v) \mathrm{d} v$,

where $A^{\Sigma}(K)$, the evolved phase space area intercepted by $\Sigma$ is simply

$A^{\Sigma}(K)=\int_{\Sigma} \mathrm{d} \xi_{x} \mathrm{~d} \eta_{x}$.

Similarly, the density of $m_{S}= \pm \frac{1}{2}$ atoms at the region of interest is given by

$\rho_{1 \pm}^{\Sigma}=\frac{1}{4 \pi} \frac{\rho_{1}^{0}}{2} \frac{R_{M}^{4}}{\Sigma} \int_{0}^{\infty}\left[A^{\Sigma}(K)\right]^{2} K^{2} F_{v^{2}}(v) \mathrm{d} v$.

It should be noted that the phase space procedure we have just outlined is rigorously applicable only in Cartesian coordinates. For beam geometries containing circular cross sections, such as those in our hydrogen beam line, the circular apertures must be bounded from below be inscribed squares and from above by circumscribed squares, the former leading to a lower limit for $\rho_{1 \pm}^{\Sigma}$ and the latter, to an upper limit.

We applied these Cartesian phase space procedures to our beam line and obtained the spectral transmittance values $\Omega_{+}(K)$ shown in Fig. 18(a) for atoms reaching the interaction region. As can be seen by comparing the phase space result with the function $\Omega_{+}(K)$ obtained from the optical model, also shown in Fig. 18(a), the agreement between the two methods is quite good. In view of the approximations made for the circular apertures, however, the phase space methods suffers considerably in accuracy, as consequence of which the phase space values of $\bar{s}$ and $\bar{\rho}$ are of diminished significance.

\section{Results and conclusions}

Following the experimental procedures described in Sect. III, we investigated the polarization, density and dissociation fraction of the hydrogen beam, and as a corollary, several calibration parameters of the Vacuum Generators Supavac quadrupole mass analyzer (QMA) as well. The determination of both the lowfield polarization, $P(0)$, and the high-field state selection parameter, $s$, requires the measurement of hydrogen beam profiles by the QMA for various settings of the Stern-Gerlach magnet driving current with the QMA alternately tuned for mass-one and mass-two observation and with the rf power applied and removed from the discharge tube. In Fig. 19(a) and (b) we display two sample mass-one profiles for SternGerlach driving currents of 0 and $3 \mathrm{~A}$ respectively, both taken with full rf power applied to the discharge tube.

Under conditions of no driving current, the beam profile should be perfectly symmetric about the cen- 

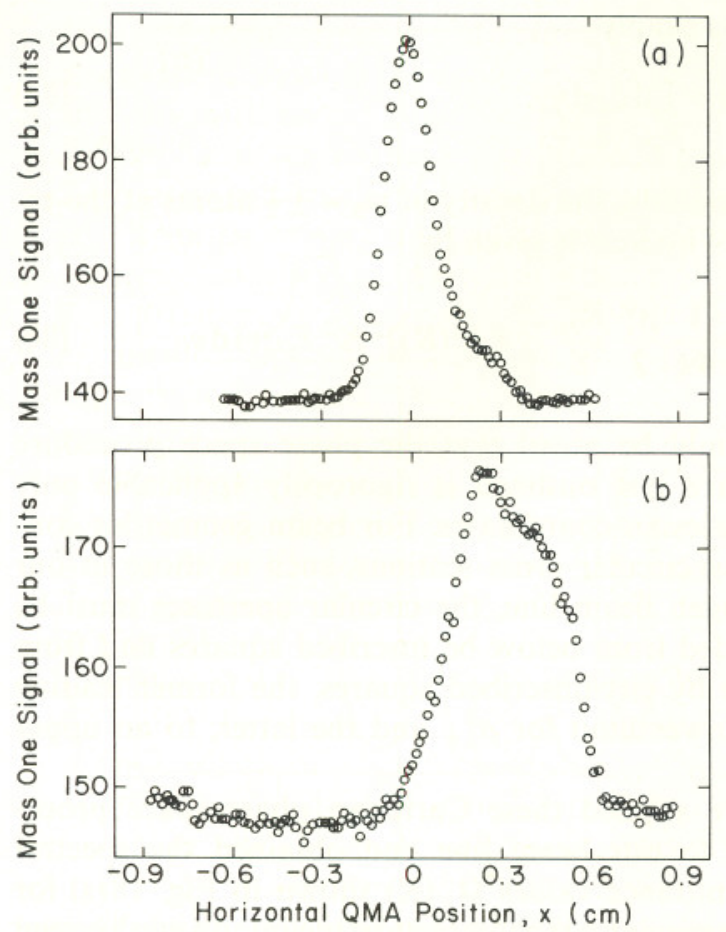

Fig. 19a and b. QMA beam profile scans carried out as described in Sect. III B. The raw data shown are for the Stern-Gerlach magnet current off in (a) and a $3 \mathrm{~A}$ driving current in (b)

troid, but as Fig. 19(a) shows the profile actually measured was asymmetric, suggesting some small degree of misalignment of either the hexapole or the SternGerlach magnet. While not of great consequence for beam operation, such a misalignment has some significance for the polarization measurement, as will become clear shortly. Examination of the profile measured for the $3 \mathrm{~A}$ driving current, illustrated in Fig. 19 (b), reveals not only an exaggerated asymmetry in the shape, as would be expected for a beam characterized by an asymmetric Maxwellian velocity distribution, but also a development of a small bump on the right side of the profile. Although reduced in prominance by slit-scattering and pole-tip scattering of the beam periphery, this bump is suggestive of one of the peaks present in the Monte-Carlo profile shown in Fig. 15(b) and no doubt is related to the chromatic properties of the hexapole magnet indicated by the characteristics of the spectral transmittance $\Omega_{+}(K)$ and the spectral transmission $\Omega_{+}(K) F_{v^{2}}(K)$ shown in Fig. 18(a) and (c) respectively.

As is evident from Fig. 19(a) and (b), the linear and quadratic terms present in the pedestal function $y^{P}(x)$ are both small, as a consequence of which the pedestals (thus dominated by a constant offset term) can be determined with good accuracy. Therefore we were able to apply the data reduction procedures summarized in Sect. III B to obtain $\Delta_{H}$ for Stern-Ger- lach driving currents between 0 and 3.5 A. At currents above $3.5 \mathrm{~A}$, scattering of the low velocity components of the beam from the pole tips of the Stern-Gerlach magnet introduces substantial uncertainties into the determination of $\Delta_{H}$. Unfortunately, as is evident from the Monte-Carlo analysis shown in Fig. 16, $\Delta_{H}$ is far from saturation at a driving current of $3.5 \mathrm{~A}$ and indeed achieves saturation only when the current reaches approximately $20 \mathrm{~A}$.

Faced with the inability to carry out measurements at saturation, we attempted to fit the measured values of $\Delta_{H}$ to those predicted by the Monte-Carlo analysis. To this end, we used standard magnetic circuit techniques in order to relate the magnetic field, $|\mathbf{H}|$, and its gradient, $\boldsymbol{\nabla} H$, in the "two-wire" geometry [15] to the applied driving current, thereby providing a common horizontal scale for the laboratory measurements and the Monte-Carlo results. We then found that the laboratory values of $\Delta_{H}$ agreed remarkably well with the on-axis Monte-Carlo values, shown in Fig. 16, provided for the former were scaled uniformly by a factor of 0.98 . Indeed this downward scaling is entirely consistent with the slight beam misalignment indicated by the asymmetric profile shown in Fig. 19(a) combined with the positional dependence of the high-field state-selection parameter, $s$.

As illustrated in Fig. 14(b), the value of $s$ at the Stern-Gerlach entrance slit, achieves a minimum of 0.975 on the beam axis and increases to more than 0.990 within a $4 \mathrm{~mm}$ horizontal offset. In other words, a small beam misalignment could easily require the application of a scale factor, of 0.985 to the measured values of $\Delta_{H}$ for purposes of comparison with the on-axis Monte-Carlo predictions. The remaining $0.5 \%$ discrepancy between the two sets of values falls easily within the experimental uncertainties. Thus we feel confident in asserting that the Monte-Carlo values of $s$ and hence $P(0)$ are verified by the experimental measurements of $\Delta_{H}$. Based upon the various uncertainties that arise in the measurements and the analyses, we conclude that at the interaction region, the hydrogen beam is characterized by the MonteCarlo values of $0.99 \pm 0.01$ for the high-field stateselection parameter $s$ and $0.515 \pm 0.005$ for the lowfield polarization $P(0)$, the precision having been slightly degraded from that of the actual Monte-Carlo results.

The validity of the Monte-Carlo modeling receives further enhancement through a comparison of the measured and predicted beam densities, $\bar{\rho}$, although experimental measurement of $\bar{\rho}$ is frought with substantial uncertainty. As explained in Sect. IIIC, $\bar{\rho}$ can be determined experimentally either by beam loading pressure measurements or alternatively by electronimpact ionization rate measurements. We carried out 
both sets of measurements and applied (22)-(25) to obtain values of $\bar{\rho}$.

For the beam loading pressure determination of $\bar{\rho}$, we make the following assumptions: effective pumping speed $S \approx 530 \mathrm{l} / \mathrm{s}$ for hydrogen molecules in the interaction chamber, as suggested by the pumping speeds and conductances given in Fig. 1; average velocity $\bar{v} \approx 6.5 \times 10^{4} \mathrm{~cm} / \mathrm{s}$, based upon an analysis of the transmission functions shown in Fig. 19(c); fraction of atoms $f \approx 0.4$ falling within $3 \mathrm{~mm}$ radius, based upon Monte-Carlo modeling; gas temperature $T \approx$ $300 \mathrm{~K}$, based upon an assumption of thermalization of molecules; and ionization gauge conversion factor of 2.2 for hydrogen molecules, based upon manufacturer's specification [40]. With these assumptions and from the measured pressure rise of $7.4 \times 10^{-9}$ Torr in the interaction chamber when the hydrogen beam is turned on we obtain a value of $6( \pm 3) \times 10^{9}$ atoms $/ \mathrm{cm}^{3}$ for $\bar{\rho}$, the large uncertainty resulting dominantly from an imprecise knowledge of the effective pumping speed for the complex pump baffling geometry used. The result is in resonable agreement with the value of $9 \times 10^{9}$ atoms $/ \mathrm{cm}^{3}$ calculated from the Monte-Carlo analysis.

For the electron impact ionization rate determination of $\bar{\rho}$ we carried out measurements at an incident electron energy of $15 \mathrm{eV}$, a value below the threshold for ionization of $\mathrm{H}_{2}$ molecules. In applying (23)-(27), we assume an electron beam radius $R_{e}$, of $1.5 \pm 0.5 \mathrm{~mm}$ and an overall experimental efficiency $\varepsilon$ of 0.10 , the latter resulting from the combination of two $90 \%$ optically transparent meshes, as shown in Fig. 2(b), and a proton impact energy of $700 \mathrm{eV}$ on the cone of a Mullard [41] channel electron multiplier for which researchers [42] have measured a detection efficiency of 0.12 with an unknown absolute precision. In light of the uncertainty in the equivalence between optical and charged particle transparency for the two meshes, and with some reasonable assumptions about the efficiency curve provided by Mullard, we assign a conservative value of \pm 0.025 to the uncertainty in $\varepsilon$.

For the total ionization cross section, $\sigma_{I}$, we use the value of $(0.762 \pm 0.038) \times 10^{-17} \mathrm{~cm}^{2}$ recently determined by Shah, Elliot, and Gilbody [43]. We note that this value is consistent with that obtained from the earlier absolute measurement of Fite and Brackman [44] at $20 \mathrm{eV}$ and scaled downward to $15 \mathrm{eV}$ in accordance with the relative measurements of McGowan and Clark [7]. With an ionization rate of 450 protons/s for an incident electron intensity of $10 \mathrm{nA}$, we obtain a value of $(1.9 \pm 0.7) \times 10^{10}$ atoms $/ \mathrm{cm}^{3}$ for $\rho_{\text {eff }}$, where we quote an overall uncertainty that includes the effect of the $\sim 100 \mathrm{meV}$ resolution (full width at half maximum) of the electron beam and the uncertainty in its energy centroid, as well as the specific uncertainties in $\varepsilon$ and $\sigma_{I}$ and to a minor degree $R_{e}$. Using the radial dependence of $\rho$ from the Monte-Carlo analysis, shown in Fig. 17, we finally obtain a value for $\bar{\rho}$ of $(1.7 \pm 0.7) \times 10^{10}$ atoms $/ \mathrm{cm}^{3}$, the slightly increased relative uncertainty arising from the uncertainty in $R_{e}$. The result again is in reasonable agreement with the Monte-Carlo value of $9 \times 10^{9}$ atoms $/ \mathrm{cm}^{3}$ and in slight disagreement with the value of $(6 \pm 3) \times 10^{9}$ atoms $/ \mathrm{cm}^{3}$ obtained from the beam loading pressure measurements.

We conclude the presentation of experimental results with a summary of the determination of the dissociation fraction at the source, $F^{0}$, defined by (31); the QMA calibration parameters $\alpha_{12} / \alpha_{22}$ and $\alpha_{11} / \alpha_{22}$ defined through (26) and (27); and the relative beam transport efficiency $\beta_{1} / \beta_{2}$ for atoms and molecules reaching the QMA from the source with the SternGerlach magnet turned off and all slits removed. Following the procedures described in Sect. IIID, we carried out measurements that under typical operating conditions lead to the following results upon the application of (26)-(40): $\quad F^{0}=0.831 \pm 0.004, \quad \alpha_{12} / \alpha_{22}$ $=0.033 \pm 0.006, \quad \alpha_{11} / \alpha_{22}=0.64 \pm 0.07, \quad$ and $\beta_{1} / \beta_{2}$ $=0.32 \pm 0.03$. Since the dissociation fraction is really quite sensitive to the actual operating conditions (pressure and rf power) of the source, we believe that the source should be characterized by a nominal dissociation fraction, $F^{0}$, between 0.8 and 0.85 .

We conclude our paper with a brief comparison of our polarized beam to hydrogen beams developed at other laboratories. As we suggested at the outset of the paper, many hydrogen beams, some polarized and some unpolarized, have indeed been constructed throughout the years, and some of these surpass our beam in one or more of their operating characteristics [11]. As a rule, however, the superior beams are far more massive and far more costly in construction and often make use of complex and expensive technologies.

In order to illustrate these points, we cite several typical examples. Employing a water-cooled $\mathrm{rf}$ $(500 \mathrm{~W}, 20 \mathrm{MHz})$ dissociator and a pair of large bore hexapole electromagnets, Dunham et al. [14] developed a polarized jet atomic beam for use as a gas target. While the density of the beam was found to be quite high, $(2-6) \times 10^{11}$ particles $/ \mathrm{cm}^{3}$, the polarization at low field, measured by $\alpha-p$ scattering, was observed to be, only $0.25-0.33$. The low value of the measured polarization is attributed to a large molecular fraction produced during the formation of the hydrogen jet.

The performance of the jet source is thus not much better than that of a more conventional one manufactured by ANAC [45], the characteristics of which 
were reported by Haeberli et al. [14] in the context of a colliding beam source for polarized ion production. The ANAC source also employs an rf dissociator and contains two large bore high field gradient hexapole magnets, one with a tapered bore and one with a fixed bore. Although achieving a high field state selection that apparently is close to unity, the ANAC source, as reported by Haeberli et al., delivers a beam whose density by contrast with the jet source is only about $8 \times 10^{10}$ atoms $/ \mathrm{cm}^{3}$ at a location approximately $25 \mathrm{~cm}$ away from the exit of the downsteam hexapole. The dissociation fraction of the ANAC source, however, can be expected to be quite high.

The design geometry of the ANAC source does not differ greatly from those of a similar source developed some years earlier by Risler et al. [12] which also uses an rf discharge $(4 \mathrm{~kW}, 27 \mathrm{MHz})$ and a large bore hexapole electromagnet containing both a tapered and a parallel section. Operating with a discharge tube pressure of $\sim 7$ Torr and employing three stages of high-speed pumping $(500 \mathrm{l} / \mathrm{s}, 50.00 \mathrm{l} / \mathrm{s}$ and $5000 \mathrm{l} / \mathrm{s}$ ), the source described by Risler et al., produces a beam density of about $8 \times 10^{10}$ atoms $/ \mathrm{cm}^{3}$ within a $10 \mathrm{~mm}$ beam diameter at a distance of $55 \mathrm{~cm}$ from the hexapole exit. No figures were quoted by Risler et al. for dissociation fraction or polarization, although both should be quite high.

Within the last few years, conventional jet beams, as well as effusive beams, have been eclipsed to some extent by the application of liquid helium technology to hydrogen atom beam production. Motivated by the well known enhancement in hexapole solid angle acceptance as the beam temperature decreases [46], the cold hydrogen sources employ "accommodators" that cool the atoms in preparation for hexapole injection. Designed for ultimate use at the Brookhaven AGS accelerator, the source developed by Herschcovitch, Kponou, and Niinikoski [15], for example, produces [47] a flux of $9.4 \times 10^{18}$ atoms sr${ }^{-1} \mathrm{~s}^{-1}$ with a most probable velocity of about $6.8 \times 10^{4} \mathrm{~cm} / \mathrm{s}$ at an accommodator temperature of $5.8 \mathrm{~K}$ or alternatively a flux of $4 \times 10^{19}$ atoms sr ${ }^{-1} \mathrm{~s}^{-1}$ with a velocity of $9.8 \times 10^{4} \mathrm{~cm} / \mathrm{s}$ at a temperature of $26 \mathrm{~K}$. While these parameters are quite impressive - at a distance of $5 \mathrm{~cm}$ from the exit of the source, the latter operating condition corresponds to an atom density of 1.6 $\times 10^{13}$ atoms $/ \mathrm{cm}^{3}$ - the cold hydrogen sources remain very much in the development stage. Use with high-field state selectors is still under investigation, and dissociation fractions are not well known.

By contrast with the complexities of helium cryogenics, the massiveness of high-gradient hexapole electromagnets, and the large investment in the use of high speed pumps, the source we have described in this paper is quite simple and inexpensive. By com- parison with other simple designs $[19,48]$, its operating parameters are very favorable and as we have shown, well understood. It is eminently suitable for small laboratory physics and with additional effort directed toward nozzle cooling can probably be improved further.

We gratefully acknowledge the financial support of the U.S. National Science Foundation (Grant PHY-8603166), the City University of New York (Grants PSC-CUNY 6-65279 and 6-67353), St. Patrick's College, and the U.K. Science and Engineering Research Council.

\section{References}

1. See for example Levy, L.P., Williams, W.L.: Phys. Rev. A 30, 220 (1984);

Hinds, E.A.: In: Spectrum of atomic hydrogen:advances. Series, G.W. (ed.), Chap. 4. Singapore: World Scientific 1987

2. See for example: Lundeen, S.R., Pipkin, F.M.: Phys. Rev. 46, 232 (1981);

Boshier, M.G., Baird, P.E.G., Foot, C.J., Hinds, E.A., Plimmer, M.D., Stacey, D.M., Swan, J.B., Tate, D.A., Warrington, D.M., Woodgate, G.K.: In: Proceedings of the Eighth International Conference on Laser Spectroscopy, Åre, Sweden, June 22-26, 1987. Persson, H., Sranberg, S.R. (eds.), p. 18. Berlin, Heidelberg, New York: Springer 1988;

Beausoleil, R.G., McIntyre, D.H., Foot, C.J., Hildum, E.A., Couilaud, B., Hänsch, T.W.: Phys. Rev. A 35, 4878 (1987)

3. Kleppner, D., Goldenberg, H.M., Ramsey, N.F.: Phys. Rev. 126, 603 (1962);

Hess, H.F., Kochanski, G.P., Doyle, J.M., Greytak, T.J., Kleppner, D.: Phys. Rev. A 34, 1602 (1986);

Hürlimann, M.D., Hardy, W.N., Berlinsky, A.J., Cline, R.W.: Phys. Rev. A 34, 1605 (1986);

4. Biraben, F., Julien, L.: Opt. Commun. 53, 319 (1985);

Hildum, E.A., Boesl, U., McIntyre, D.H., Beausolell, R.G., Hänsch, T.W.: Phys. Rev. 56, 576 (1986);

Zhao, P., Lichten, W., Layer, H.P., Bergquist, J.C.: Phys. Rev. A 34, 5138 (1986)

5. See for example Williams, J.F.: In: Electron and photon interactions with atoms. Kleinpoppen, H., McDowell, M.R.C. (eds.), p. 309. New York: Plenum 1976;

Teubner, P.J.O., Lloyd, C.R., Weigold, E.: Phys. Rev. A 9, 2552 (1974);

Lloyd, C.R., Teubner, P.J.O., Weigold, E., Lewis, B.R.: Phys. Rev. A 10, 175 (1974);

Callaway, J.F., Williams, J.F.: Phys. Rev. A 12, 2312 (1975);

Slevin, J., Eminyan, M., Woolsey, J.M., Vassilev, G., Porter, H.Q., Back, C.G., Watkin, S.: Phys. Rev. A 26, 1344 (1982);

Hood, S.T., Weigold, E., Dixon, A.J.: J. Phys. B 12, 631 (1979);

Williams, J.F.: J. Phys. B 14, 1197 (1981)

6. Fletcher, G.D., Alguard, M.J., Gay, T.J., Gughes, V.W., Wainwright, P.F., Lubell, M.S., Raith, W.: Phys. Rev. A 31, 2854 (1985)

7. McGowan, J.W., Clarke, E.M.: Phys. Rev. 167, 43 (1968)

8. Donahue, J.B., Gram, P.A.M., Hynes, M.V., Hamm, R.W., Frost, C.A., Bryant, H.C., Butterfield, K.B., Clark, D.A., Smith, W.W.: Phys. Rev. Lett. 48, 1538 (1982)

9. Slevin, J., Stirling, W.: Rev. Sci. Instrum. 52, 1780 (1981)

10. Clausnitzer, G., Fleischmann, R., Schopper, H.: Z. Phys. 144, 336 (1956)

11. Excellent summaries of the status of high-intensity polarized hydrogen beams as applied to polarized sources and targets for accelerator applications may be found in the Proceeding of the 
International Workshop on Polarized Sources and Targets. Jaccard, S., Mango, S. (eds.): Helv. Phys. Acta 59, 513 (1986); and in: Polarized proton ion sources. Roy, G., Schmor, P. (eds.). AIP Conf. Proc. No. 117, American Institute of Physics, New York, 1984. For a comparison of earlier work on ground state sources, see Glavish, H.F.: Proceedings of the Symposium on Ion Sources and Formation of Ion Beams, October 19-21, 1971, Brookhaven National Laboratory, p. 207. BNL Report No. 50310

12. Risler, R., Grüebler, W., Konig, V., Schmelzbach, P.A.: Nucl. Instrum. Methods 121, 425 (1974)

13. Parker, E.F.: In: High-Energy Physics with Polarized Beams and Targets, Argonne, 1976. AIP Conf. Proc. No. 35. Marshak, M.L. (ed.), p. 382. American Institute of Physics, New York, 1977 Mavis, D.G., Dunham, J.S., Hugg, J.W., Glavish, H.F.: ibid., p. 517

14. Haeberli, W., Barker, M.D., Gossett, C.A., Mavis, D.G., Quin, P.A., Sowinsky, J., Wise, T., Glavish, H.F.: Nucl. Instrum. Methods 196, 319 (1982);

Dunham, J.S., Galovich, C.S., Glavish, H.F., Hanna, S.S., Mavis, D.G., Wissink, S.W.: Nucl. Instrum. Methods 219, 46 (1984)

15. Walraven, J.T.M., Silvera, I.F.: Rev. Sci. Instrum. 53, 1167 (1982) hershcovitch, A., Kponou, A., Niinikoski, T.O.: Rev. Sci. Instrum. 58, 547 (1987)

16. McKibben, J.L., Lawrence, G.P., Ohlsen, G.G.: In: Polarization Phenomena in Nuclear Reactions, Proceedings of the Third International Symposium, Madison 1970. Barschall, H.H., Haeberli, W. (eds.), p. 828. University of Wisconsin Press, Madison 1971; Preiskchat, E., Michel, G., Roth, G.W., Cramer, J.G., Jr., Weitkamp, W.G.: ibid., p. 832;

Clegg, T.B., Bissinger, G.A., Haeberli, W., Quin, P.A.: ibid., p. 835 ;

Donnally, B.L.: ibid., p. 295

17. The literature is rich with descriptions of such work. For a survey of some recent applications, the reader is referred to the proceedings of a number of specialized conferences such as High Energy Physics with Polarized Beams and Polarized Targets, Proceedings of the 1980. Joseph, C., Soffer, J. (eds.). Basel: Birkhäuser 1981;

High-Energy Spin Physics, Brookhaven 1982, AIP Conf. Proc. No. 95, Bunce, G.M. (ed.), American Institute of Physics, New York 1983;

Intersections Between Particle and Nuclear Physics, AIP Conf. Proc. No. 123, Mischke, R.R. (ed.), American Institute of Physics, New York 1984;

Polarized Beams at SSC \& Polarized Antiprotons, AIP Conf. Proc. No. 145, Krisch, A.D., Lin, A.M.T., Chamberlain, O. (eds.), American Institute of Physics, New York, 1986; Proceedings of the Sixth International Symposium on Polarization Phenomena in Nuclear Physics, Osaka 1985; J. Phys. Soc. Jpn. [Suppl.] 55, $1062(1986)$

18. Wood, R.W.: Proc. R. Soc. 97, 455 (1920); 102, 1 (1922)
19. Shah, M.B., Gilbody, H.B.: J. Phys. B 14, 2361 (1981)

20. Alguard, M.J., Clendenin, J.E., Ehrlich, R.D., Hughes, V.W., Ladish, J.S., Lubell, M.S., Baum, G., Raith, W., Miller, R.H., Lysenko, W.: Nucl. Instrum. Methods 163, 29 (1979)

21. Ramsey, N.F.: Molecular beams. London: Oxford University Press 1963

22. Innotec Group, Inc. (formerly Torr Vacuum Products) Model S-1000

23. Balzers Model TPU 200

24. Perkin-Elmer Model 207-0284

25. Perkin-Elmer Model 207-0164

26. CTI Cryogenics Model Cryo Torr 7

27. Leisk Engineering

28. Ziock, K.P., Little, W.A.: Rev. Sci. Instrum. 58, 557 (1987)

29. Baum, G.: Private communication;

Weber, M.: Aufbau und Erprobung eines spinpolarisierten $\mathrm{He}\left(2^{3} \mathrm{~S}\right)$-Atomstrahls mit umpolbarer Polarisationsrichtung. Diplomarbeit, Fakultät für Physik, Universität Bielefeld (unpublished)

30. See for example Hughes, V.W., Long, R.L., Jr., Lubell, M.S., Posner, M., Raith, W.: Phys. Rev. A 5, 195 (1972)

31. Kusch, P., Hughes, V.W.: In: Handbuch der Physik. Flügge, S. (ed.), Vol. 3711, p. 1. Berlin: Springer 1959

32. Audoin, C., Desaintfuscien, M., Schermann, J.P.: Nucl. Instrum. Methods 69, 1 (1969)

33. Kessler, J.: Polarized electrons, 2nd Edn. Berlin, Heidelberg, New York: Springer 1985

34. Bevington, P.: Data reduction and error analysis for the physical sciences. New York: McGraw Hill 1969

35. Roth, A.: Vacuum technology, 2nd Edn. Amsterdam: North Holland 1982

36. Although an alternate definition of dissociation fraction, $\left(\rho_{1}^{0}\right) /\left(\rho_{1}^{0}+2 \rho_{2}^{0}\right)$, is often used, we prefer the definition given by Eq. 45 since it is more directly analogous to the fraction of events related to atoms as obtained in crossed beams experiments (see Ref. 6)

37. Lew, H.: In: Methods of experimental physics. Hughes, V.W., Schultz, H.L. (eds.), Vol. 4/A, p. 155. New York: Academic Press 1967

38. Ladish, J.S.: Production of polarized electrons. Ph.D. Thesis, Yale University, 1974

39. English, T.C., Gallagher, T.F.: Rev. Sci. Instrum. 40, 1484 (1969)

40. Granville-Phillips Model 274-023 ionization gauge

41. Mullard Model X919 BL

42. Timothy, A.F., Timothy, J.G.: Acta Electronica 14, 159 (1971)

43. Shah, M.B., Elliott, D.S., Gilbody, H.B.: J. Phys. B 20, 3501 (1987)

44. Fite, W.L., Brackman, R.T.: Phys. Rev. 112, 1141 (1958)

45. ANAC Model No. 2909

46. Haeberli, W.: Ann. Rev. Nucl. Sci. 17, 373 (1967)

47. Hershcovitch, A.: Private communication

48. Hood, S.T., Dixon, A.J., Weigold, E.: J. Phys. E 11, 948 (1978) 